\title{
Exploration of Saint-Venant's principle in inertial high strain rate testing of materials
}

\author{
Haibin $\mathrm{Zhu}^{1,2}$, Fabrice Pierron ${ }^{2}$ \\ ${ }^{1}$ LASMIS Université de Technologie de Troyes, 12 rue Marie Curie, CS 42060, 10004, Troyes cedex, France \\ ${ }^{2}$ Faculty of Engineering and the Environment, University of Southampton, Southampton SO17 1BJ, UK
}

\begin{abstract}
Current high strain rate testing procedures of materials are limited by poor instrumentation which leads to the requirement for stringent assumptions to enable data processing and constitutive model identification. This is the case for instance for the well known Split Hopkinson Pressure Bar (SHPB) apparatus which relies on strain gauge measurements away from the deforming sample. This paper is a step forward in the exploration of novel tests based on time and space resolved kinematic measurements obtained through ultra-high speed imaging. The underpinning idea is to use acceleration fields obtained from temporal differentiation of the full-field deformation maps measured through techniques like Digital Image Correlation (DIC) or the grid method. This information is then used for inverse identification with the Virtual fields Method. The feasibility of this new methodology has been verified in the recent past on a few examples. The present paper is a new contribution towards the advancement of this idea. Here, inertial impact tests are considered. They consist of firing a small steel ball impactor at rectangular free standing quasi-isotropic composite specimens. One of the main contributions of the work is to investigate the issue of through-thickness heterogeneity of the kinematic fields through both numerical simulations (3D finite element model) and actual tests. The results show that the parasitic effects arising from non-uniform through-the-thickness loading can successfully be mitigated by the use of longer specimens, making use of Saint-Venant's principle in dynamics.
\end{abstract}

Key words: Virtual Fields Method; High strain rates; Inertial effects; Full-field measurements; Grid method

\section{Introduction}

Fibre-reinforced composite materials are currently used in many applications where they are potentially submitted to high strain rate events (runway debris impact on aircraft panels, crash of automotive components etc.). The mechanical behaviour of these materials at high rates is known to be significantly different from that under static loading. Therefore, it is essential to investigate and to characterise the strain rate dependent behaviour of these materials with a view to structural design and numerical simulation. Over the years, different strategies for characterising the mechanical behaviour of materials at high strain rates have been developed in the scientific community. A review of the main experimental techniques for high rate tests is available in [1]. Among these techniques the most popular is the so-called the Split Hopkinson Pressure Bar (SHPB) or Kolsky bar. The original idea was proposed a century ago by Hopkinson [2], while the current split bars system was designed by Kolsky [3]. In the recent decades the SHPB technique has been applied for high strain rate testing of a number of materials. The review paper [1] has many citations on the use of the SHPB, for instance. However, the SHPB procedure suffers a number of shortcomings. First, it is based on the one-dimensional wave theory; therefore, it strictly relies on the assumption of uniaxial and

Email addresses: haibin.zhu@soton.ac.uk (Haibin $\mathrm{Zhu}^{1,2}$ ), f.pierron@soton.ac.uk (Fabrice Pierron ${ }^{2}$ ) 
homogeneous stress state. Then, another stringent assumption is the fact that the standard SHPB analysis based on the strain gauges readings on the input and output bars requires quasi-static loading conditions, i.e., no inertial effects. As a consequence, specimens usually have to be very short to minimize the time over which stress waves travel back and forth within the specimen and fade away. This is even worse for materials with low sound speeds like soft materials and biological tissues. Although some authors proposed improvements to address some of these issues $[4,5]$, the process of reconstructing the wave and load histories still requires some very constraining assumptions.

One of the reasons for the limitations of the SHPB procedure is the rather poor instrumentation it relies on. Only a few strain gauges are used to analyse the complex behaviour of a material submitted to a high rate impact. Unlike conventional strain measurement techniques such as extensometers or strain gauges, full-field measurement techniques such as digital image correlation (DIC) [6] and the grid method [7] provide much richer experimental information. It is therefore natural to expect that this new wealth of information will lead to an in-depth revisit of the high strain rate testing procedures. In the past few years, DIC has been used to acquire full-field information in the SHPB tests [8-10]. However, full-field deformation measurements were mainly used to provide average strains over a certain area like a non-contact strain gauge, which did not take full advantage of the strain imaging capability to measure nominally heterogeneous strain states.

Using a more complex test configuration has consequences on how material parameters can be extracted. Away from the restrictive assumptions of the classical SHPB analysis, there is no more direct link between the strain measurements and the material parameters to be identified. As a consequence, it is necessary to resort to some so-called 'inverse resolution'. A number of strategies (e.g. the Finite Element Model Updating (FEMU) method [11], the Virtual Fields Method (VFM) [12, 13], etc.) based on full-field measurements have been proposed in the past. It is beyond the scope of the present paper to recall the details of these methods, information can be found in the following review paper [14]. FEMU has been applied in the past to identify the constitutive parameters of materials from full-field measurements at high strain rates [15]. However, in that work, the cost function used to identify the model parameters only included the measured and simulated impact forces, also meaning that the assumptions necessary to obtain the force from the strain gauge readings on the input bar still had to hold. In particular, wave dispersion and inertial effects had to be neglected. These assumptions are not always true at high strain rates, especially for specimens with complex shapes or made from soft materials [16, 17]. A more recent study [18] combined impact force and strain at the centre of the specimen to construct the cost function but again, inertial effects were neglected and force measurements relied on specific hypotheses. Finally, strain maps have been used in FEMU on a SHPB test [19] but again, the force needed to be measured with a Hopkinson bar. It should also be noted that all these studies were looking at rather large plastic strains where inertial effects were not a problem anymore. To the best knowledge of the present authors, such a FEMU methodolgy has not been applied to materials in the elastic range.

An alternative to the popular FEMU technique is the Virtual Fields Method (VFM) [13]. In a nutshell, the VFM uses the full-field measurements to derive stresses using an assumed constitutive model and identifies the parameters of this model by checking stress equilibrium using the global (or weak) form of stress equilibrium known as the principle of virtual work. The main benefit from this is that iterative solving of finite element models is avoided, resulting in much faster computations. This is even more critical at high strain rates where a single finite element model may already take several to several tens of minutes to run and this will have to be done many times to evaluate the cost function until convergence, leading to several hours of computations until convergence $(2.5$ to $3 \mathrm{~h}$ in [19] for one parameter only, private communication from the corresponding author, J. Kajberg).

The present paper makes use of the VFM to extract the constitutive parameters from the full-field kinematic measurements. Here, only linear elastic behaviour will be considered as a first step towards more complex constitutive models. Linear elasticity is also one of the most challenging situations as the strains are small and inertial effects significant when not predominant. In quasi-static situations, it is necessary to measure a load information in order to identify stiffness components. Otherwise, only stiffness ratios can be obtained. In dynamics however, the acceleration field due to inertial effects can be used as an alternative load cell provided that the density of the material is known. This important underpinning concept has already been demonstrated in the recent past [20-22]. It has great potential to change in depth the way 
high strain rate testing is performed in the future.

The current work complements the three already published contributions cited above. First, it relies on purely inertial loading and as a consequence, presents more challenges in virtual fields definition than [22]. The special optimized virtual field approach already reported in [21] is also used here but in the present case, the test configuration is designed to lead to a more heterogeneous state of stress. Indeed, while the line impact employed in [21] led to a mainly unidirectional stress state, the ball impact configuration in the present work generates a more complex stress and strain state and is therefore one step further towards test complexity. Finally, one of the important novelties of the present work compared to [21] is the systematic numerical and experimental study of the effects of non-uniformity of the kinematic fields through the thickness and the suggestions for ways to mitigate these effects. Full 3D finite element models have been developed and specimens with different lengths have been tested in order to investigate the applicability of Saint-Venant's principle in such dynamic inertial tests.

In the present paper, three quasi-isotropic composite specimens with different lengths were impacted by a small ball-bullet. The microsecond-level events were recorded by a digital ultra-high speed (UHS) camera (Shimadzu HPV-X). The grid method was used to perform full-field deformation measurements at high strain rates. The experimental procedure and measurement performance are introduced in Section 2 . The VFM with inertial effects is then recalled in Section 3. 3D simulations of the ball impact tests including the misalignment of the contact point and the effect of specimen length are provided in Section 4. Experimental results and related identification using different methodologies are detailed in Section 5.

\section{Experimental procedure}

\subsection{Tests and materials}

The specimens tested in this paper were laminated to the following $[0 / 45 /-45 / 90] s$ stacking sequence from CYTEC MTM58FRB carbon/epoxy prepreg. The thickness of the specimens is around $3.7 \mathrm{~mm}$. The nominal quasi-static stiffness parameters are: $E_{11}=124 \mathrm{GPa}, E_{22}=7.5 \mathrm{GPa}, \nu_{12}=0.31, G_{12}=4.0 \mathrm{GPa}$ [23]. Due to the nature of the quasi-isotropic layup, the in-plane elastic stiffness components of the specimens only depend on two parameters. Based on lamination theory, the in-plane Young's modulus of this quasiisotropic laminate is $47.1 \mathrm{GPa}$ and Poisson's ratio is 0.31. Additionally, the specimens are expected to exhibit low strain rate dependence because the behaviour of this quasi-isotropic lay-up is highly dominated by the fibres which do not show any significant strain rate dependence. Therefore, the quasi-static Young's modulus and Poisson's ratio will be used as the reference values in this paper. However, for a unidirectional layup, the strain rate dependence of the material properties proved quite significant, especially on the transverse and shear moduli $[24,25]$. The extension of the present work to unidirectional specimens is currently underway and will be reported in the near future.

The experimental set-up used to perform the impact tests is shown in Figure 1. It is composed of a gun connected to an air pump and a $9 \mathrm{~mm}$ diameter steel ball used as the projectile. The specimen was positioned in front of the gun with the help of a foam support. A thin steel tab of thickness $1 \mathrm{~mm}$ was bonded onto the impact end of the specimen so as to mitigate the stress concentration. This has the unfortunate effect of also absorbing some of the impact energy as the tab deforms plastically but tests without the tabs have proved worse with too much damage to the composite specimens at the impact end, which is detrimental to the present analysis. This is clearly a disadvantage of the ball impact when compared to the planar impact used in [21]. The fired ball and specimen were both enclosed in a chamber with transparent perspex walls at its top and front faces. Three quasi-isotropic specimens with different lengths but same width were tested in this study. The dimensions and the impact speeds are shown in Table 1.

\subsection{Ultra-high speed imaging}

To record the impact event, a Shimadzu HPV-X UHS camera was used in this work. The imaging configuration is shown in Table 2. This camera uses a dedicated sensor called FTCMOS which is a special type of CMOS sensor with on-board solid-state memory storage. More details about this camera can be found in $[26,27]$. 
The images of the deforming specimens were acquired by the camera through a transparent PMMA window. The selected frame rate was 5 million frames per second ( fps). Due to the short inter-frame time, two strong flash lights (Bowens Prolite 60, here) proved necessary. The output power of each flash light is 300 Joules. The rising time is around 50 microseconds and the flash duration is 1 millisecond. In order to acquire high quality images and to make full use of the recording capacity of the camera, the images should be acquired with full flash intensity, and the camera should be triggered at the instant the steel ball contacts with the specimen. Thus, two independent triggering systems are necessary. The triggering system used here consisted in two closing circuits. One was positioned between the exit of the gun barrel and the specimen and consisted of a piece of metallic wire wound around a cardboard frame and a thin metal strip fixed at the back of the frame. When the ball passed through the frame, it pushed at least one of the wires against the metal strip, closing the circuit and triggering the flash lights. Since the distance between the frame and the front end of the specimen was about $15 \mathrm{~mm}$, this ensured that the imaging took place roughly midway through the lighting event. The other triggering system consisted of two small pieces of wire attached to the front end of the specimen but separated by about $1 \mathrm{~mm}$. When the ball reached the specimens, it made contact with both wires, closing the circuit and triggering the camera. This was not $100 \%$ accurate since before impacting the specimen, the ball had first to crush the wires but thanks to the built-in post-triggering of the camera, satisfactory triggering was achieved.

\subsection{The grid method}

Although Digital Image Correlation (DIC) is currently the most popular full-field strain measurement technique, an attractive alternative for small heterogeneous strain distributions is the grid method, which has recently been used in high rate dynamics testing [20, 21]. This technique presents a slightly better compromise between spatial resolution and strain resolution at the cost of bonding a grid onto the test piece. This is the technique that has been selected here.

The grid method is a full-field optical technique measuring displacement $[7,28]$. A grid pattern consisting of a set of horizontal and vertical contrasted lines was transferred onto the surface of the specimen using an appropriate procedure [29]. The details for the grid method is not introduced here in order not to overload the manuscript. The reader is referred to [20,30]. During the tests, the grid did not peel or flake off. Comparison with strain gauge data in [20] showed good fidelity of the grid measurements in dynamic.

From the displacement vector, strain components can be computed by spatial differentiation. Correspondingly, for fully time-resolved displacements in dynamics, acceleration fields can be calculated through second order differentiation over time from the displacements. Similarly, strain rate fields can be obtained through first order differentiation over time from the time-resolved strains. However, these numerical differentiations amplify the noise contained in the measured displacement [31]. Therefore, spatial and temporal smoothing are usually necessary for strain, strain rate and acceleration calculation.

\subsection{Resolution}

Before moving to the dynamic tests, it is necessary to assess the accuracy of the deformation measurements. For full-field measurements, the resolution can be evaluated as the standard deviation of deformation maps obtained from stationary images. In this work, it has been qualified by capturing images of the stationary specimen just before the tests, using the same imaging conditions as in the impact tests. Theoretically, the displacement, strain and acceleration between two nominally stationary images should be uniformly null, but in practice, this is not the case because of the digital noise. Therefore, the displacement should be spatially smoothed before differentiation. To perform spatial smoothing, different methods are available, e.g. polynomial fitting [32, 33], diffuse approximation [34, 35], Gaussian filter [36, 37], etc. For temporal smoothing, a local least-squares algorithm can be applied on a sliding window with fixed number of images to reconstruct the displacement and strain in the form of a polynomial function, for instance. In this work, spatial smoothing was performed with a Gaussian filter, see Table 2. The acceleration and strain rate fields were calculated from the displacement and strain fields respectively using temporal smoothing. The smoothing parameters (size of sliding window and Gaussian smoothing kernel) have been selected after a convergence study to find an appropriate compromise between resolution and spatial resolution. It is beyond 
the scope of the present paper to investigate the effect of these parameters. This will be performed in the future using the simulation simulator developed in [30, 38]. Average standard deviations of displacement, strain and acceleration over all frames for specimen 1 are presented in Table 2 . The performances were similar for the other two specimens.

Comparing the information in Table 2 with that in [21], it can be seen that the measurement performances are very similar, except for a slightly better acceleration resolution in the present work. This shows that the measurement performance is quite robust for this particular camera. The dynamic images have been smoothed using the same configuration to extract full-field strain and acceleration maps. Finally, these full-field data are processed by the VFM which is recalled in the next section.

\section{The Virtual Fields Method}

The Virtual Fields Method is based on the principle of virtual work which in dynamics and in absence of body forces can be expressed as [21]:

$$
-\int_{V_{m}} \underline{\underline{\sigma}}: \underline{\underline{\epsilon}} \underline{\underline{t}}^{*} d V+\int_{\partial V_{m}} \vec{T} \cdot \vec{u}^{*} d S=\int_{V_{m}} \rho \frac{\partial^{2} \vec{u}}{\partial t^{2}} \cdot \vec{u}^{*} d V
$$

where $\underline{\sigma}$ represents the Cauchy stress tensor, $\vec{T}$ the Cauchy stress vector acting at the boundary surface $\partial V_{m}$, $\vec{u}^{*}$ a $C^{\overline{0}}$ vectorial function referred to as 'virtual displacement field', $\underline{\epsilon}^{*}$ the virtual strain tensor derived from $\vec{u}^{*}$ and $\rho$ the density of the material. '?' denotes the scalar product between vectors whereas ':' represents the contracted product between matrices (or scalar product for matrices). In case of an in-plane test on a thin test piece, if $h$ is the thickness of the volume $V_{m}$ and $S$ the specimen planar surface, Equation 1 reduces to a $2 D$ situation with:

$$
-h \int_{S} \underline{\underline{\underline{\sigma}}}: \underline{\underline{\epsilon}} \underline{\epsilon}^{*} d S+h \int_{\partial S} \vec{T} \cdot \vec{u}^{*} d L=h \int_{S} \rho \frac{\partial^{2} \vec{u}}{\partial t^{2}} \cdot \vec{u}^{*} d S
$$

To identify the constitutive parameters, a non-parametric approach was proposed by Othman et al. $[39,40]$ consisting in calculating the average stress in any transverse slice of a rectangular specimen loaded uniaxially in a Kolsky bar from a measured force at the boundary, the full-field acceleration maps and the cross-sectional area of the specimen. The stress-strain curve can then be obtained using the full-field strain maps. In fact, the internal virtual work in Equation 2 can be zeroed out through a rigid virtual field (see [21] for the details of the derivation), leading to:

$$
\overline{\sigma_{x}(x, t)}=\rho x \overline{a_{x}(x, t)}
$$

where $t$ is the time, $\overline{\sigma_{x}}$ the average longitudinal stress over the transverse slice at a given $x$ coordinate, and $\overline{a_{x}}$ the spatial average of the longitudinal acceleration component over the area between the free end and the slice at coordinate $x$.

With the reconstructed stress above and the corresponding average strain calculated from the measured strain over the same transverse slice, local stress-strain curves can be plotted for each frame. In this way, the elastic modulus can be extracted from these curves without the need for any a priori constitutive model, hence the term 'non-parametric'. Another two sets of virtual fields were proposed in [21] to reconstruct shear stress and moment. This non-parametric approach is simple but may not necessarily work in all situations. In the more general case, a parametric VFM approach needs to be employed.

In Equation 2, $\vec{T}$ represents the stresses applied at the boundary of the solid. In the present case, if the whole specimen is considered, this vector is only non zero at the right-hand side boundary and represents the impact loading. It is not easy to measure external forces at high rates because of inertial effects. However, it is possible to define a particular virtual field to cancel out the virtual work of the impact forces. To do so, 
the virtual displacement components along the right-hand side boundary of the region of interest in Figure 1 can be set to zero. Thus, Equation 2 can be rewritten as:

$$
-\int_{S} \underline{\underline{\sigma}}: \underline{\underline{\epsilon}}^{*} d S=\int_{S} \rho \frac{\partial^{2} \vec{u}}{\partial t^{2}} \cdot \vec{u}^{*} d S
$$

This equation is valid for any virtual fields provided that the virtual displacement vector is continuous and null at the boundary where unknown impact forces are acting. Since there are two unknown parameters here, only two independent virtual fields are required to identify them. The virtual fields can be defined manually as described in [22]. However, this paper will make use of the special optimised virtual fields proposed in $[41,42]$. This is an automated procedure to define virtual fields minimizing the effect of noise on the identified parameters. Moreover, this procedure provides ratios between the standard deviations and mean values of the identified stiffness components. These ratios indicate the noise sensitivity of each identified stiffness component. In the present work, bilinear finite elements have been used to expand the virtual fields. The bilinear elements ensure displacement continuity between elements, as needed for the principle of virtual work. More details can be found in [13, 42].

\section{Finite element simulation}

\subsection{FE simulation of offset contact}

Series of $3 D$ finite element models of the impact tests have been built up. The objective of these simulations was to investigate the influence of $3 D$ wave propagation on the identification of parameters and to search for an effective way mitigate these effects. It is worth emphasising that only the full-field data at the top and bottom surfaces were processed, because this is the information available experimentally. However, the average fields over the thickness were output as well and used as reference in the following. This $3 D$ model is composed of an isotropic specimen of dimensions $40 \times 30 \times 4 \mathrm{~mm}$ (length $\times$ width $\times$ thickness) impacted by a steel ball of diameter $9 \mathrm{~mm}$. The input Young's modulus was $47.5 \mathrm{GPa}$, and 0.3 for the Poisson's ratio. The dynamic response was simulated using ABAQUS/EXPLICIT to produce fullfield strain and acceleration maps which were then processed by the VFM. The details of the FE simulations are shown in Table 3. Both temporal and spatial convergence has been checked. From this simulation, it was found that the contact time between the two solids was about 20 microseconds. Moreover, damping proved necessary in the computations to improve numerical stability. In FE simulations, classical Rayleigh damping was considered [43]. It combines the mass and stiffness damping through the mass-proportional and stiffness-proportional coefficients $\alpha$ and $\beta$, respectively. For instance, Figure 2 plots the stress-strain curves at $x=20.75 \mathrm{~mm}$ with different damping parameters, from Equation 3. One can clearly see that, without damping ( $\alpha=0 s^{-1}$ and $\beta=0 s$ ), the curve is linear only when the two solids are in contact, whereas after the contact phase, the data are inconsistent. With large damping (e.g. $\alpha=0 s^{-1}$ and $\beta=1.10^{-6} s$ ), this model experiences significantly non-linear behaviour, as expected for a visco-elastic material. Therefore, the damping coefficient should be small enough to respect the condition of elastic material behaviour but large enough to damp the numerical instabilities during computation. It can be seen from Figure 2 (c) that damping coefficients of $\alpha=0 s^{-1}$ and $\beta=2 \cdot 10^{-8} s$ are appropriate, because the curve throughout the impact simulation is linear. Therefore, this set of values will be kept for the simulations presented in this paper. It is also worth noting that the current approach enabling to calculate stress from acceleration is also a relevant method to check for the quality of dynamic explicit computations.

A first item to investigate is the effect of the point load. Indeed, this generates stress and strain states that are not uniform through the thickness, as opposed to the cylindrical impactor configuration used in [21]. This will generate an error when transforming the volume integrals in Equation 1 into surface integrals in Equation 2. Moreover, in practice, perfect centring of the point contact in the cross-section is not easy to achieve. For instance, Figure 3 shows the photos of the tested specimens and tabs in the experimental tests. One can clearly see that the contact point is not always perfectly centred. Aside from small discrepancies of the test specimen position in the impact chamber, it is thought that the wires used for triggering may 
also cause some slight deflection of the ball at the moment of impact. This may result in an even larger error when estimating the volume integrals from surface measurements. In order to investigate these issues, three contact models have been considered. Contact model (1) describes an ideal case where the full-field data at the top and bottom surfaces are symmetrical about the mid-plane. Contact model (2) represents a small misalignment of $0.5 \mathrm{~mm}(12.5 \%$ of the thickness $)$ from the mid-plane in the cross-section, and a larger misalignment of $1.5 \mathrm{~mm}$ (37.5\% of the thickness) is considered in contact model (3). Due to this misalignment, the stress wave propagation in the specimen tends to be three-dimensional. It is thought however that the metal tab used to protect the specimen can already act as a load spreader to mitigate this effect. This was considered in the present simulations but only with elastic behaviour for the tab in order to save computation time.

The ideal contact model (1) without the steel tab was simulated first. The strain and acceleration fields at the top and bottom surfaces were output. The average fields over the thickness were also output and used as the reference field as this is the data that will provide exact estimations of the surface integrals in Equation 2 from the volume integrals in Equation 1. The difference between the top surface strain and the average strain through the thickness in the longitudinal direction at 14 microseconds is shown in Figure 4 (a). The strain difference represents about $10 \%$ of the global strain values. One can clearly see a wave pattern produced by the multiple reflections of the waves on the top and bottom surfaces. The strain concentrations caused by the point load appear very clearly. The heterogeneity of the mechanical fields is far more pronounced than for the quasi-uniaxial case in [21]. Figure 4 (b) shows the same data but with the tab. It is interesting to see that the curvature of the strain ripples is less pronounced with the tab, showing the load spreading effect of the tab. The top to average difference is also slightly smaller than without the tab, as expected. Finally, one can see that away from the loading point, the error fades away, illustrating a kind of Saint-Venant effect $[44,45]$ in dynamics. In practice however, the metal tab exhibits local yielding as seen on Figure 3, thus absorbing some of the impact energy that will not be available to deform the specimen. Nevertheless, the tab will prevent local indentation damage in the material which is beneficial for the analysis presented here. Therefore, $1 \mathrm{~mm}$ thick steel tabs will systematically be used in the rest of the paper, for both simulations and experiments.

Contact model (2) with the tab was then simulated. The differences in longitudinal strain at the top and bottom surfaces at 14 microseconds are shown in Figure 5(a). It can be seen that the strains are much more heterogeneous through the thickness. This problem will significantly disrupt the identification results as the volume integrals will be falsely evaluated from the surface ones. In addition to the tabs, another interesting idea to mitigate these $3 D$ effects is to lengthen the specimen in order to be in a better situation to benefit from Saint-Venant's principle. In this case, the field of view will be restricted to $40 \mathrm{~mm}$ from the free-end side of the specimen to be consistent with experimental conditions as imaging a longer field of view would compromise the spatial resolution of the measurements. Two models with specimen lengths of 60 and $80 \mathrm{~mm}$ for contact models (2) and (3) respectively were simulated with the same FE configuration as for the $40 \mathrm{~mm}$ specimen. Figure 5(b) and (c) presents the differences in longitudinal strains at the top and bottom surfaces for the two longer specimens at different time steps. Comparing three sets of maps in Figure 5, one can clearly see that for the two longer specimens the strain differences at both surfaces are significantly lower than that for the specimen of length $40 \mathrm{~mm}$, although the average strains through the thickness of the three specimens are comparable. This means that lengthening the specimen mitigates the through-thickness strain heterogeneity in the specimen.

\subsection{Identification from simulated data}

This section presents three different procedures to extract the elastic stiffness parameters from the simulated data, with increasing complexity. The first one assumes Poisson's ratio as known and investigates the identification of $E$ through the plots of stress-strain curves. The second one brings this one step further by using over-determined systems arising from the application of Equation 3. Finally, the full Virtual Fields Method approach is considered. 


\subsubsection{Stress-strain curves from simulated data}

The influence of misalignment can also be quantitatively verified by the non-parametric method described in Section 3. Figure 6 presents the average longitudinal stress as a function of the average $\varepsilon_{x}+\nu \varepsilon_{y}(\nu=0.3$, here) at $x=20.75 \mathrm{~mm}$ from the free end. The slope of the stress-strain curve provides the stiffness component $Q_{x x}$ which related to $E$ through:

$$
\left\{\begin{array}{c}
E=Q_{x x}\left(1-\nu^{2}\right) \\
\nu=Q_{x y} / Q_{x x}
\end{array}\right.
$$

It is clear that the stress-strain curves from the top and bottom surfaces for contact model (1) are consistent with each other and linear, and the estimated Young's modulus for model (1) is around 47.2 GPa, very close to the input value of $47.5 \mathrm{GPa}$. For contact model (2) however, the curves from the top and bottom surfaces diverge, although the curve from the average data through the thickness matches that of contact model (1) very well, as expected.

To validate the Saint-Venant effect in the dynamic simulation, the stress-strain curves at $x=20.75 \mathrm{~mm}$ from the free end for all offset contact models mentioned previously are presented in Figure 7. It can clearly be seen that longer specimens lead to reduced discrepancies in the stress-strain curves from the top and bottom surfaces, even though larger misalignment was considered for the specimen of length $80 \mathrm{~mm}$. The estimated $E$ through fitting the first 20 points of the stress-strain curve on the top surface of the longest specimen is about $47.7 \mathrm{GPa}$. Young's modulus can also be identified for all transverse slices of the $40 \mathrm{~mm}$ long field of view along the longitudinal axis of the specimen. Figure 8 presents the identified Young's modulus for all offset contact models. It seems that, for all specimens, the identified results from the top and bottom surfaces are symmetric about the reference value and tend to converge at the free (left) end. Moreover, with the increase of specimen length, the discrepancies of the identified Young's modulus from the top and bottom surfaces are reduced, even though a larger offset of the point contact was considered for the longest specimen. This means that lengthening the specimen reduces the stress/strain heterogeneity through the thickness at slices away from the impact. This solution was implemented experimentally to check for its practical validity.

\subsubsection{Over-determined system solution from simulated data}

According to Hooke's law, the relationship between stress and strain in the longitudinal direction can be expressed as:

$$
\sigma_{x}=Q_{x x} \varepsilon_{x}+Q_{x y} \varepsilon_{y}
$$

In this work, $Q_{x x}$ and $Q_{x y}$ in Equation 6 are unknown. The stress and strain components in this equation can also be expressed in the form of average values. The average longitudinal stress can be constructed by the acceleration at each transverse slice as in Equation 3, and the strains averaged over the same slice. Experimentally, the acceleration and strain fields can be calculated from the displacement through temporal and spatial numerical differentiations respectively. In this FE simulation, they were output from ABAQUS directly (here, 50 data frames were output). Combining Equations 3 and 6, the following relationship is obtained:

$$
\rho x \overline{a_{x}(x, t)}=Q_{x x} \overline{\varepsilon_{x}}+Q_{x y} \overline{\varepsilon_{y}}
$$

This equation can be used for each transverse slice at all times when strain and acceleration maps are available. So, at each section, an over-determined system consisting of 50 equations (from the 50 data frames) with two unknowns $Q_{x x}$ and $Q_{x y}$ can be built up. This can be solved for $Q_{x x}$ and $Q_{x y}$ by a least-squares solution. However, for all offset contact models, the linearity of the stress-strain curves is only restricted to the loading stage. The reason for this is currently unknown but might be caused by erroneous FE calculation. In any case, in the rest of this paper, all of the over-determined system solutions only consist of data from the loading stage. 
It must be emphasised that for contact model (1), Young's modulus is calculated through Equation 5 with the identified Poisson's ratio, whereas for contact models (2) and (3), $E$ is calculated with $\nu=0.3$ due to the inaccurate identification of Poisson's ratio. Firstly, the identified $E$ and $\nu$ at each slice for the short specimen with contact model (1) are plotted in Figure 9. For the good contact model, it is clear that the identification of Young's modulus is very good, except for the identification at slices close to the free and impact ends. This is not surprising for the free end as the stress values become very small. For the impact end, one would expect errors for the top and bottom data but not for the average. This is probably caused by errors in the FE data arising from the impedance difference between the steel tab and the tested material. As for the identification of Poisson's ratio, it is not as good as Young's modulus. For the offset contact models, the identified results are presented in Figure 10. It can be seen that the identification is significantly worse than that for contact model (1). For the same specimen, the identified Young's modulus from the top and bottom surfaces converges at the left part of the specimen. This tendency is consistent with that in Figure 8, however, the identification from the over-determined system is worse than that in Figure 8. The exact reason for this has not been established yet but it might be that at some time during the loading, each slice contains a low stress and low strain situation which provides unstable lest-square inversion. This will need to be investigated in the future as this identification approach is very appealing because of its simplicity.

It is also possible to consider all slices at the same time to identify one overall value of $E$ and $\nu$. Since it has been shown that the data close to the impact and free ends are not reliable, only a central section away from the ends has been kept by discarding 4 and 12 columns of data from the free and impact ends respectively over a total of 80 columns of data. The identified overall Young's modulus and Poisson's ratio are shown in Table 4. The percentages in brackets represent the errors from the reference. It is clear that for all contact models the identified results from the average fields through the thickness are good. Moreover, the identification of Poisson's ratio is worse than that of Young's modulus. For the offset contact models (2) and (3), the identification is unsatisfactory, however, with increasing specimen length, the errors reduce, even for the larger offset considered for the longest specimen.

\subsubsection{VFM identification from simulated data}

The same simulated sets of data were processed by the VFM. Firstly, the data from the whole field of view were considered. In this procedure, the virtual mesh used to expand the virtual fields is composed of 4 elements in the $x$-direction and 3 elements in the $y$-direction. The virtual displacement along the right-hand side boundary of the field of view is set to 0 so as to cancel out the virtual work of the impact forces at the right end. In the VFM, $Q_{x x}$ and $Q_{x y}$ are first identified. Young's modulus and Poisson's ratio are then calculated through Equation 5. The results for contact model (1) are shown in Figure 11. It can be seen that the identified results from the top and bottom surfaces match, even though they slightly diverge from that from the average data through the thickness, even for the good contact model. Moreover, the identified Young's modulus from the top and bottom surfaces are significantly higher than that for the average data during the first 20 microseconds (within the contact stage), thereafter, they gradually converge to the reference value. This was expected because, for the good contact model, during the contact stage the strain levels on the top and bottom surfaces are always lower than the average value through the thickness, which leads to higher Young's modulus on the top and bottom surfaces. When contact is lost at around 20 microseconds, the strain state through the thickness tends to be more uniform, hence the converged values. In addition, as seen in Figure 11, some oscillations can clearly be observed at around 20 and 30 microseconds, especially for Poisson's ratio. It is still not clear what is causing this.

It has been shown that the identification at slices close to the free and impact ends of the specimen is not reliable, as seen in Figures 8 and 10. In the VFM processing, if only data from the central area is considered (the same data for large over-determined system in Section 4.2.2), the VFM results in Figure 12 are obtained. It is worth noting that in this case the virtual displacement vector along the left and right boundaries of the region of interest is necessarily set to zero so as to cancel out the virtual work of the unknown forces applied at both boundaries. As seen in Figure 12, it is clear that the identification errors are significantly reduced, and the results from the surfaces and the through-thickness average match very well. This is mainly because the through-thickness stress and strain heterogeneities are concentrated at the 
impact end, as already illustrated previously. However, some oscillations at 20, 30 and 40 microseconds persist and cannot be interpreted at the present time.

The simulated data for the offset contact models have also been processed with the VFM. The data over the complete field of view was kept here, since as the results were much worse than for contact model (1), removing the end data did not significantly change the trends of the results. As mentioned previously, for the offset contact models, good linearity of stress-strain curves is only restricted to the loading stage. Moreover, in practice, with the current imaging configuration, the recording duration is only 25.6 microseconds. Therefore, for all offset contact models, only the identification within the first 25 microseconds is presented in the following. The identification for the short specimen $(L=40 \mathrm{~mm})$ proved quite unsatisfactory, as shown in Figure 13. It is clear that the modulus identified from the top surface data is systematically lower than that from the bottom surface. The reason is the same as for the results in Figure 11. On the top surface, strains are larger than on the bottom surface within the first 25 microseconds for the short specimen, hence the identified Young's modulus at the top surface is lower. Figure 14 shows the VFM identification results for the longer specimens. It can be seen that the discrepancies of the identified Young's Modulus and Poisson's ratio are significantly reduced through lengthening of the specimen, even though a larger offset has been considered for the longest specimen. However, for these long specimens, the identification from the top surface seems to be higher than that from the bottom surface, which is opposed to the trend seen in Figure 13. This is thought to be caused by an inversion of the through-thickness strain distribution pattern as the wave propagates further down the longer specimens.

As a conclusion to this section on numerical simulations, all three identification strategies have confirmed that increasing the specimen length mitigates the effect of through-thickness strain heterogeneity and provides results closer to the reference. The next section is dedicated to an experimental investigation of this effect.

\section{Experimental results}

\subsection{Full-field deformation results}

Three tests with different quasi-isotropic composite specimens were performed on the impact rig as shown in Figure 1. The dimensions of the specimens and the impact speeds are presented in Table 1. The acquired images were processed according to the configurations presented in Table 2. For specimen $1(L=40 \mathrm{~mm})$, the maps of strain and acceleration at 9 microseconds with respect to the triggering of the camera are shown in Figure 15. It is worth emphasising that for this specimen, the surface used for data processing has been reduced from the image field of view by removing $6 \mathrm{~mm}$ from the impact end of the specimen in order to avoid the strain distortion due to the permanent damage caused by the localized impact point. It can be seen that these fields are symmetric or antisymmetric (for the shear components) with respect to the horizontal axis of the specimen, as expected. This confirms that the impact point is positioned at the middle of the impacted surface in the $y$ direction. Only elastic deformation was considered in the FE analysis whereas experimentally, tab plasticity and composite damage occur at the loading point. As shown in Figure 3, significant delamination at the impact end of specimen 3 was observed, in spite of the steel tab. This dissipates a significant amount of energy and leads to lower strain and acceleration levels.

The full-field maps for specimens 2 and 3 are shown at 13 microseconds in Figure 16 and at 17 microseconds in Figure 17 respectively. Different times were selected for the three specimens to show comparable strain and acceleration maps as the triggering time varied between tested specimens. As seen in these figures, longer specimens lead to more uniaxial and unidirectional strain and acceleration maps. This was expected as part of Saint-Venant's principle acting in the $(x, y)$ plane.

Figure 18(a) shows the temporal evolution of the average of the longitudinal strain over the field of view. As seen in this figure, the strain profiles are consistent with the impact speeds reported in Table 1, as the $40 \mathrm{~mm}$ specimen was impacted at a lower ball speed. In this work, the strain levels are only about a tenth of that reported in [21]. Complete wave rebounds are captured except for specimen 3 due to late triggering. The complete set of dynamic maps are provided as 'Supplementary material' to this article. The next part of this section is dedicated to the processing of these data for stiffness identification purposes. 


\subsection{Simplified VFM identification}

The approaches used in Section 4 for the simulated data are applied to the identification from experimental data as well. Firstly, Young's modulus is extracted from stress-strain curves at each transverse slice along the longitudinal axis of the specimen. For instance, stress-strain curves from different specimens at $x=17.7 \mathrm{~mm}$ are shown in Figure 18(b). The impact speed for specimen 1 was lower than for the other two specimens, as already mentioned previously, explaining the 'truncated' stress-strain curve. The stress-strain curves for the three specimens are reasonably linear and consistent with each other. It is worth noting that there are significant oscillations for specimen 2 and 3 marked by black circles. Figure 19(a) presents the profiles of displacement, velocity and acceleration for specimen 2 during the test. Even though the displacement curve is very smooth, a first differentiation to obtain velocity shows slight disturbances (magnification window). This is further enhanced by the next differentiation to obtain acceleration. The disturbance is now clearly visible, and will translate to the stresses obtained from Equation 3. This can be traced back to some artefacts in the imaging. The mean intensity profile of the raw images of specimen 2 during this dynamic test is shown in Figure 19(b). One can see an increase of the mean intensity as a function of time. This is a characteristic of the camera sensor and happens systematically. Fortunately, the phase extraction is not sensitive to the mean image intensity so this did not affect the displacement measurements. However, some oscillations can be observed there, especially early in the image series, as marked by the red circle. This is similar to the problem reported for the earlier version of this camera, the Shimadzu HPV-1 [46], although on a much smaller scale. Clearly here, the temporal smoothing used to derive acceleration reduces this effect but does not cancel it. Nevertheless, its impact on the global stress-strain curve is rather limited, as seen on Figure 18(b).

Young's modulus for each transverse slice along the longitudinal axis of the specimen is shown in Figure 20(a). One can clearly see that at slices close to both specimen ends, the results are not good. This trend is similar to that from the simulated data in Section 4. The estimated Young's modulus over the central part of the field of view is comparatively good. Figure 20(b) reports the goodness of fit (R-square values) for all slices for each specimen. This figure first confirms that only a certain distance away from both ends is the behaviour linear, as already evidenced before. Moreover, the plot for specimen 1 shows oscillations certainly related to the significantly $3 D$ nature of wave propagation, resulting in more perturbed stress-strain curves. Specimens 2 and 3 both exhibit excellent fit at a higher level than specimen 1, confirming the relevance of using longer specimens to mitigate for the $3 D$ stress wave propagation. It is also interesting to note that for both specimens 2 and 3, the identified Young's modulus is systematically larger than the value identified in quasi-static tests. This was not expected after the numerical results which showed unbiased estimation for $E$. However, these numerical simulations used the strains and accelerations directly from the FE model whereas as noted before, the full-field measurements provide significant low-pass filtering (both temporally and spatially) of the data. Temporal smoothing reduces the acceleration levels whereas spatial smoothing decreases the strain peaks. Since $E$ results from a balance between two terms containing these data, positive or negative systematic errors can certainly be obtained. The only way to investigate this further would be to simulate the image processing as in the simulator presented in [30] but this is beyond the scope of the present paper.

As seen in Figure 20, the identification in the central section of the field of view proved more stable. Therefore, a large over-determined system consisting of data in the central section has been built up frame by frame and used to identify the overall Young's modulus and Poisson's ratio. According to the identification in Figure 20, different regions of interests were selected. The details are shown in Table 1. The identified results are shown in Table 5 . It is clear that the identified $\nu$ is very bad. Thus, Young's modulus is calculated with $\nu=0.3$. The modulus from specimens 2 and 3 is closer to the expected reference, in spite of the large contact point offset for specimen 3, as seen in Figure 3. This illustrates again the benefit of longer specimens.

\subsection{Full VFM identification}

The same data used to build up the large over-determined system was also processed by the VFM. In this case, the virtual displacement components along the left and right boundaries of the region of interest are necessarily set to zero so that the virtual work of the unknown forces applied at both boundaries can be 
zeroed out. Concerning the virtual mesh, in FE simulations it is not critical as the data are (nearly) exact. However, the experimental data is noisy and different virtual fields provide different results. Following a convergence study on the virtual mesh density, the virtual mesh selected here is composed of 13 elements in the $x$-direction and 3 elements in the $y$-direction. The identified Young's modulus and Poisson's ratio are presented in Figure 21. Here again, Young's modulus has been calculated with $\nu=0.3$. The identified values for $E$ are consistent with the results from the over-determined system. The values are systematically lower for the $40 \mathrm{~mm}$ specimens and systematically higher for the other two, to about the same levels. Poisson's ratio was also reasonably estimated, particularly for the $60 \mathrm{~mm}$ specimen.

Finally, it is interesting to look at the ratios between the standard deviations and mean values of the identified stiffness components, denoted $\eta_{x x} / Q_{x x}$ and $\eta_{x y} / Q_{x y}$ in this paper and provided by the optimized virtual field procedure. The lower the $\eta / Q$ values, the better the identification. As such, these parameters provide an indication of the quality of the identification. High values of these ratios indicate poor signal to noise ratios for the identification [42].

The values of these parameters are shown in Figure 22. As can be seen, the values of $\eta_{x x} / Q_{x x}$ and $\eta_{x y} / Q_{x y}$ for specimen 1 are systematically higher than for the other two specimens. This is consistent with the lower strain levels for this test, as seen previously. Also, the abnormally high values at the early and late stages are because of low signal to noise ratios, as explained before. Finally, it is clear that the levels of $\eta_{x y} / Q_{x y}$ are higher than that of $\eta_{x x} / Q_{x x}$ for the three specimens. This is not surprising as Poisson's ratio has less influence on the actual strain field than Young's modulus and is always going to be more difficult to identify.

Generally, the identified results are close to the quasi-isotropic reference values and the previous results reported in [21]. This is even more remarkable if one considers the very low strain levels involved here, systematically less than 1000 microstrains, together with the more complex $2 D$ strain distribution arising from a point load and the $3 D$ parasitic effects coming from the non-uniform through-thickness load distribution. This strengthens the fact that this new inertial testing approach already presented in a few papers in the past has great potential to become a practical tool for high strain rate identification in the future, even though plenty of work is still to be done to realize this potential.

\section{Conclusion and perspectives}

This paper has presented a new contribution towards the long term goal of defining novel high strain rate material testing procedures based on high speed full-field kinematic measurements and inverse identification with the virtual fields method. The underpinning novelty is the use of the full-field acceleration maps as a volume distributed load cell, avoiding the need for external impact force measurement. Compared to a previous feasibility study published in [21], much smaller strain levels were recorded in the present work, providing a good test case for the robustness of the identification method. The main conclusions of the present paper are as follows.

- The new point load impact configuration gives rise to heterogeneous distributions of stress and strain through the thickness which evolve in time and space as the waves propagate and bounce off the different specimen faces.

- The presence of a thin $1 \mathrm{~mm}$ steel tab slightly mitigates this problem, though its plastic deformation absorbs a significant amount of the impact energy which led to small experimental strain levels in the specimen.

- Using longer specimens and a field of view containing the free end away from the impact point, the $3 D$ effects can be sufficiently mitigated to obtain good mechanical identification. As such, this study has confirmed the existence of a Saint-Venant effect in high rate dynamics, as already established by previous authors and reported in [47], with a fade away distance of around one to two times the width.

- Experimental results on three different specimen lengths confirmed the findings of the simulations: longer specimens provided more stable and precise identification. 
- Finally, the new results presented here confirmed that the present alternative to classical Split Hopkinson Pressure Bar has potential to become a standard technique in the future.

Clearly, this is still early days for this new procedure and much more work is needed to make it fully operational as a routine testing technique. First, the identification is only elastic here. While this is justified at these early stages to validate the technique and test its robustness, the real interest lies in non-linear behaviour. Such inertial impact tests have already been conducted on off-axis unidirectional specimens and the results will be reported soon. Extension to elasto-visco-plasticity for metals is also underway and in the near future, more materials and constitutive models will be considered to widen the applicability of the technique. Finally, it will be necessary to delve into test configuration design by adapting recent tools developed for quasi-static situations [30] to such inertial impact tests.

\section{Acknowledgements}

This work is supported by China Scholarship Council (CSC) through the government grant of Haibin Zhu. Professor Pierron gratefully acknowledges support from the Royal Society and the Wolfson Foundation through a Royal Society Wolfson Research Merit Award. The authors would like to acknowledge Mr Brian Speyer from Speyer Photonics Ltd and Dr. Markus Ortlieb from Shimadzu Europa GmbH for lending the camera and helping out with the experiments. They would also like to thank Dr. Nicola Symonds and Dr. Liam Goodes from the nCATS group at the University of Southampton for their help with the impact rig.

\section{References}

[1] J. Field, S. Walley, W. Proud, H. Goldrein, C. Siviour, Review of experimental techniques for high rate deformation and shock studies, International Journal of Impact Engineering 30 (7) (2004) 725-775.

[2] B. Hopkinson, A method of measuring the pressure produced in the detonation of high explosives or by the impact of bullets, Philosophical Transactions of the Royal Society A 213 (1914) 437-456.

[3] H. Kolsky, An investigation of the mechanical properties of materials at very high rates of loading, Philosophical Transactions of the Royal Society B 62 (1949) 676-700.

[4] R. Gerlach, C. Kettenbeil, N. Petrinic, A new split Hopkinson tensile bar design, International Journal of Impact Engineering 50 (2012) 63-67.

[5] S. Mousavi, K. Welch, U. Valdek, B. Lundberg, Non-equilibrium split Hopkinson pressure bar procedure for non-parametric identification of complex modulus, International Journal of Impact Engineering 31 (9) (2005) 1133-1151.

[6] M. Sutton, S. McNeill, J. Helm, J. Yuh, Advances in two-dimensional and three-dimensional computer vision, Photomechanics, Topic Applied Physics (2000) 323-372.

[7] Y. Surrel, Fringe analysis, Photomechanics, Topics Applied Physics 77 (2000) 55-99.

[8] A. Gilat, T. Schmidt, A. Walker, Full field strain measurement in compression and tensile split Hopkinson bar experiments, Experimental Mechanics 49 (2) (2008) 291-302.

[9] H. Koerber, J. Xavier, P. Camanho, High strain rate characterisation of unidirectional carbon-epoxy IM7-8552 in transverse compression and in-plane shear using digital image correlation, Mechanics of Materials 42 (11) (2010) 1004-1019.

[10] H. Luo, H. Lu, N. Leventis, The compressive behavior of isocyanate-crosslinked silica aerogel at high strain rates, Mechanics of Time-Dependent Materials 10 (2) (2006) 83-111.

[11] P. Ienny, A. Caro-Bretelle, E. Pagnacco, Identification from measurements of mechanical fields by finite element model updating strategies, European Journal of Computational Mechanics 18 (3-4) (2009) 353-376.

[12] M. Grédiac, F. Pierron, S. Avril, E. Toussaint, The virtual fields method for extracting constitutive parameters from full-field measurements: a review, Strain 42 (4) (2006) 233-253.

[13] F. Pierron, M. Grédiac, The Virtual Fields Method: extracting constitutive mechanical parameters from full-field deformation measurements, Springer New-York, 2012.

[14] S. Avril, M. Bonnet, A. Bretelle, M. Grédiac, F. Hild, P. Ienny, F. Latourte, D. Lemosse, S. Pagano, E. Pagnacco, F. Pierron, Overview of identification methods of mechanical parameters based on full-field measurements, Experimental Mechanics 48 (4) (2008) 381-402.

[15] J. Kajberg, K. Sundin, L. Melin, P. Ståhle, High strain-rate tensile testing and viscoplastic parameter identification using microscopic high-speed photography, International Journal of Plasticity 20 (4-5) (2004) 561-575.

[16] S. Grantham, C. Siviour, W. Proud, J. Field, High-strain rate Brazilian testing of an explosive simulant using speckle metrology, Measurement Science and Technology 15 (5) (2004) 1867-1870.

[17] B. Song, Dynamic stress equilibration in split Hopkinson pressure bar tests on soft materials, Experimental Mechanics 44 (3) (2004) 300-312.

[18] J. Peirs, P. Verleysen, W. Van Paepegem, J. Degrieck, Determining the stress-strain behaviour at large strains from high strain rate tensile and shear experiments, International Journal of Impact Engineering 38 (2011) 406-415. 
[19] J. Kajberg, B. Wikman, Viscoplastic parameter estimation by high strain-rate experiments and inverse modellingspeckle measurements and high-speed photography, International Journal of Solids and Structures 44 (1) (2007) 145-164.

[20] F. Pierron, P. Forquin, Ultra-high-speed full-field deformation measurements on concrete spalling specimens and stiffness identification with the virtual fields method, Strain 48 (5) (2012) 388-405.

[21] F. Pierron, H. Zhu, C. Siviour, Beyond Hopkinson's bar, Philosophical Transactions of the Royal Society A 372 (2023) (2014) $1-24$.

[22] R. Moulart, F. Pierron, S. Hallett, M. Wisnom, Full-field strain measurement and identification of composites moduli at high strain rate with the virtual fields method, Experimental Mechanics 51 (4) (2011) 509-536.

[23] M. Longana, Intermediate strain rate testing methodologies and full-field optical strain measurement techniques for composite materials characterisation, PhD thesis, University of Southampton (2014).

[24] H. Hsiao, I. Daniel, R. Cordes, Strain rate effects on the transverse compressive and shear behavior of unidirectional composites, Journal of Composite Materials 33 (17) (1999) 1620-1642.

[25] M. Shokrieh, M. Omidi, Investigation of strain rate effects on in-plane shear properties of glass/epoxy composites, Composite Structures 91 (1) (2009) 95-102.

[26] Y. Tochigi, K. Hanzawa, Y. Kato, R. Kuroda, H. Mutoh, R. Hirose, H. Tominaga, K. Takubo, Y. Kondo, S. Sugawa, A global-shutter CMOS image sensor with readout speed of 1-Tpixel/s burst and 780-Mpixel/s continuous, IEEE Journal of Solid-State Circuits 48 (1) (2013) 329-338.

[27] Y. Kondo, K. Takubo, H. Tominaga, R. Hirose, N. Tokuoka, Y. Kawaguchi, Y. Takaie, A. Ozaki, Development of "HyperVision HPV-X" high-speed video camera, Shimadzu Review (2013) SR3-003E.

[28] Y. Surrel, Moiré and grid methods: a signal processing approach, Photomechanics 2342 (1994) $118-127$.

[29] J. Piro, M. Grédiac, Producing and transferring low-spatial-frequency grids for measuring displacement fields with moiré and grid methods, Experimental Techniques 28 (4) (2004) 23-26.

[30] M. Rossi, F. Pierron, On the use of simulated experiments in designing tests for material characterization from full-field measurements, International Journal of Solids and Structures 49 (3-4) (2012) 420-435.

[31] M. D'Amico, G. Ferrigno, Technique for the evaluation of derivatives from noisy biomechanical displacement data using a model-based bandwidth-selection procedure, Medical \& Biological Engineering \& Computing 28 (5) (1990) $407-415$.

[32] G. Wood, Data smoothing and differentiation procedures in biomechanics, Exercise and Sport Sciences Reviews 10 (1982) 308-362.

[33] F. Pierron, B. Green, M. Wisnom, Full-field assessment of the damage process of laminated composite open-hole tensile specimens. Part I: methodology, Composites Part A: Applied Science and Manufacturing 38 (11) (2007) 2307-2320.

[34] S. Avril, P. Feissel, F. Pierron, P. Villon, Estimation of the strain field from full-field displacement noisy data. Comparing finite elements global least squares and polynomial diffuse approximation, European Journal of Computational Mechanics 17 (5-7) (2008) 857-868.

[35] S. Avril, P. Feissel, F. Pierron, P. Villon, Comparison of two approaches for differentiating full-field data in solid mechanics, Measurement Science and Technology 21 (1) (2010) 015703.

[36] R. Haddad, A. Akansu, A class of fast Gaussian binomial filters for speech and image processing, IEEE Transactions on Acoustics, Speech and Signal Processing 39 (1991) 723-727.

[37] Digital filters: Gaussian smoothing, http://homepages.inf.ed.ac.uk/rbf/HIPR2/gsmooth.htm.

[38] M. Rossi, P. Lava, F. Pierron, D. Debruyne, M. Sasso, Effect of DIC spatial resolution, noise and interpolation error on identification results with the VFM, Strain In press.

[39] S. Aloui, R. Othman, A. Poitou, P. Guégan, S. El-Borgi, Non-parametric identification of the non-homogeneous stress in high strain-rate uni-axial experiments, Mechanics Research Communications 35 (6) (2008) 392-397.

[40] R. Othman, S. Aloui, A. Poitou, Identification of non-homogeneous stress fields in dynamic experiments with a nonparametric method, Polymer Testing 29 (5) (2010) 616-623.

[41] E. Toussaint, M. Grédiac, F. Pierron, The virtual fields method with piecewise virtual fields, International Journal of Mechanical Sciences 48 (3) (2006) 256-264.

[42] S. Avril, M. Grédiac, F. Pierron, Sensitivity of the virtual fields method to noisy data, Computational Mechanics 34 (6) (2004) 439-452.

[43] I. Chowdhury, S. Dasgupta, Computation of Rayleigh damping coefficients for large systems, The Electronic Journal of Geotechnical Engineering 8 (2003) Bundle C.

[44] Y. Arimitsu, N. Kazumi, S. Toyomitsu, A study of Saint-Venant's principle for composite materials by means of internal stress fields, Journal of Applied Mechanics, Transactions ASME 62 (1) (1995) 53-58.

[45] F. Pierron, Saint-Venant effects in the Iosipescu specimen, Journal of Composite Materials 22 (1998) $1986-2015$.

[46] M. Rossi, F. Pierron, P. Forquin, Assessment of the metrological performance of an in situ storage image sensor ultra-high speed camera for full-field deformation measurements, Measurement Science and Technology 25 (2) (2014) 025401.

[47] B. Karp, D. Durban, Saint-Venant's principle in dynamics of structures, Applied Mechanics Reviews 64 (2) (2011) 020801. 


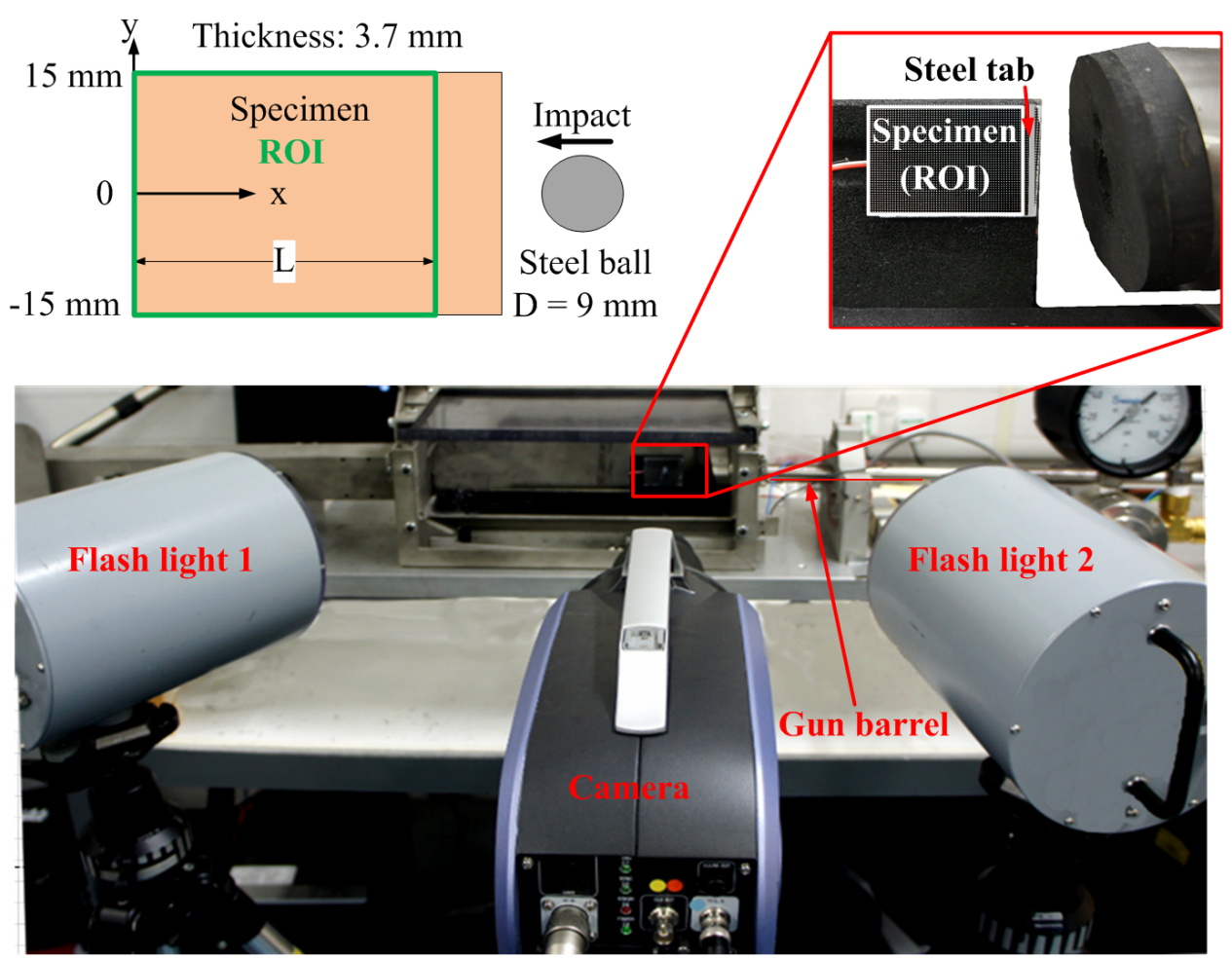

Figure 1: Schematic and picture of the impact test set-up. 


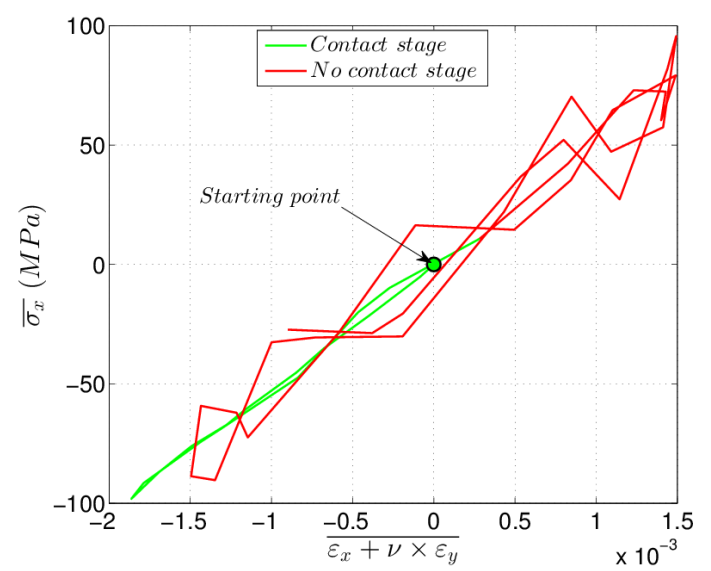

(a)

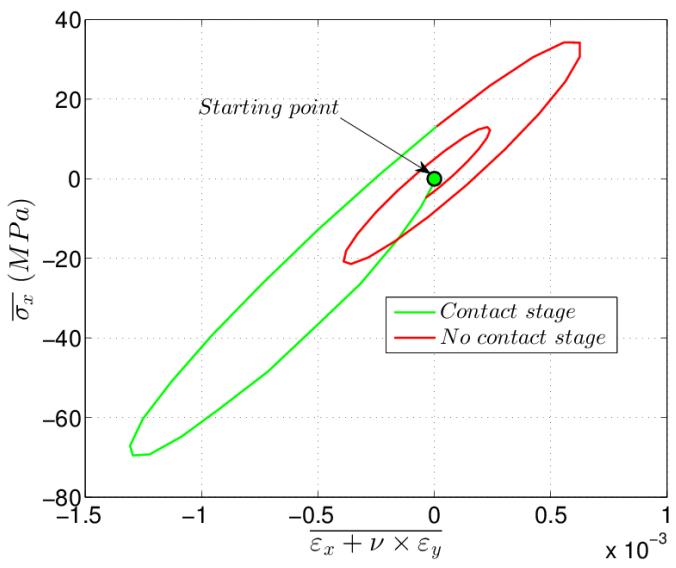

(b)

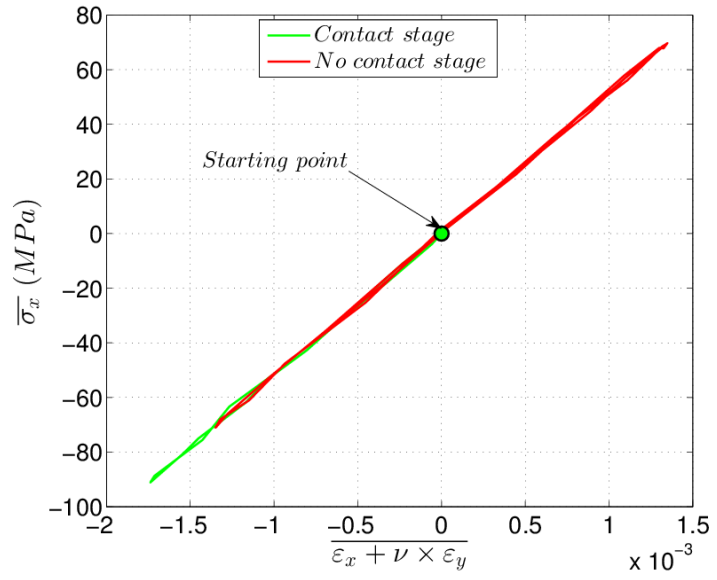

(c)

Figure 2: Stress-strain curve from data at the top surface at $x=20.75 \mathrm{~mm}$ with different damping parameters. Contact duration: 20 microseconds. (a) $\alpha=0 s^{-1}, \beta=0 s$. (b) $\alpha=0 s^{-1}, \beta=1.10^{-6} s$. (c) $\alpha=0 s^{-1}, \beta=2.10^{-8} s$. 


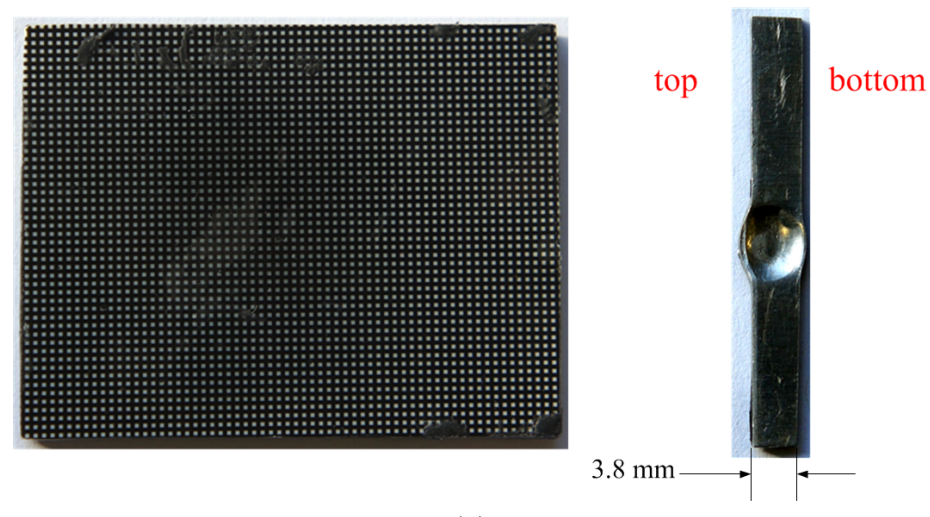

(a)
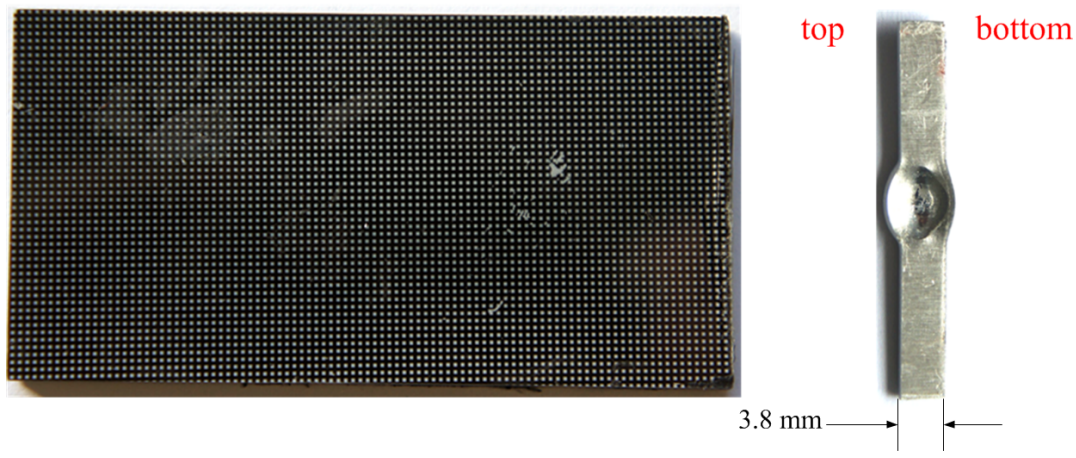

(b)

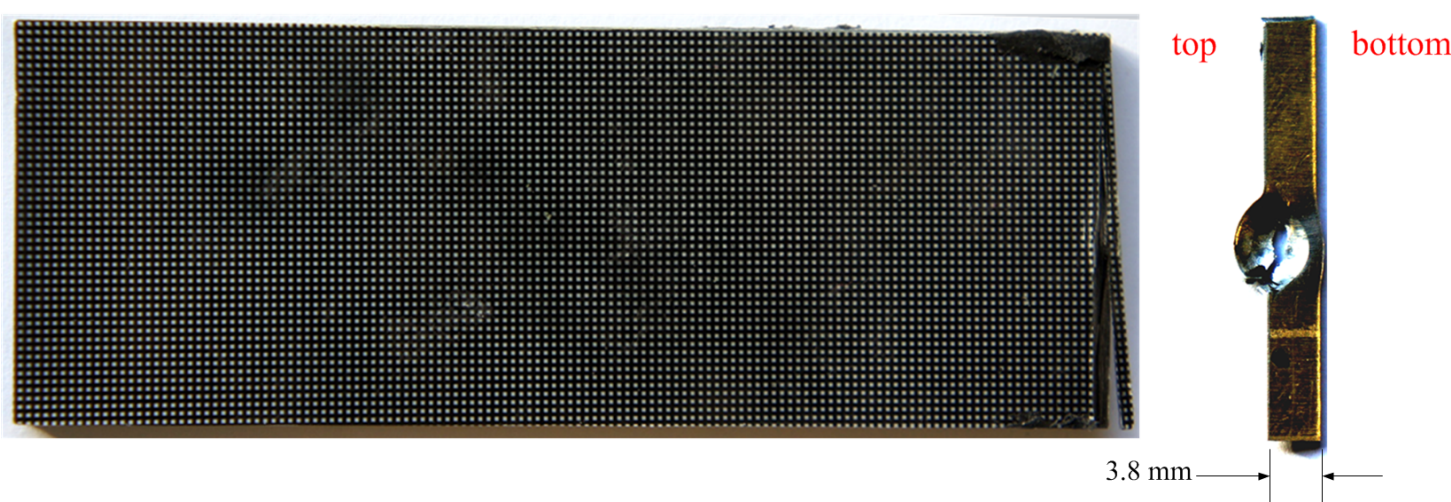

(c)

Figure 3: Pictures of the tested specimens and associated steel tabs. (a) Specimen 1, misalignment: $1 \mathrm{~mm}$ (b) Specimen 2, misalignment: $0.5 \mathrm{~mm}$. (c) Specimen 3, misalignment: $1.5 \mathrm{~mm}$ 

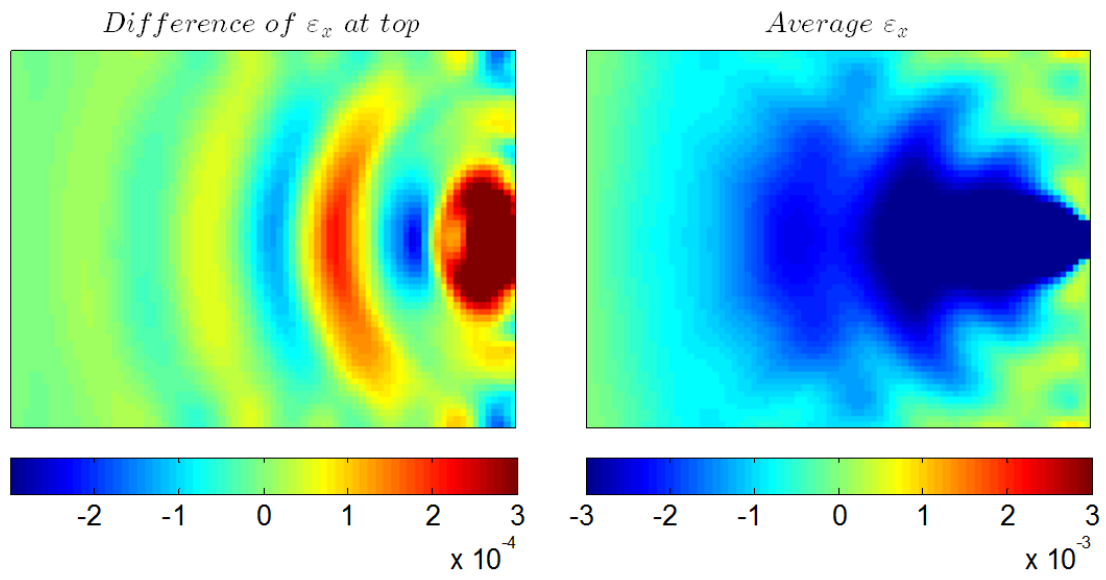

(a)
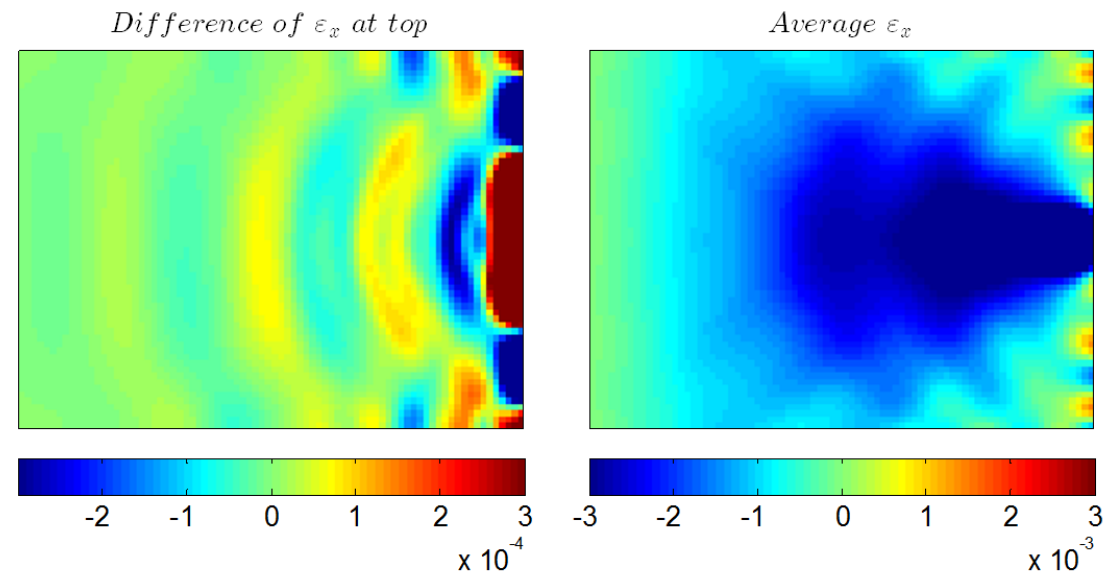

(b)

Figure 4: Differences between the top surface and through-thickness average strains in the longitudinal direction at 14 microseconds. (a) Without tab. (b) With tab. $L=40 \mathrm{~mm}$. 

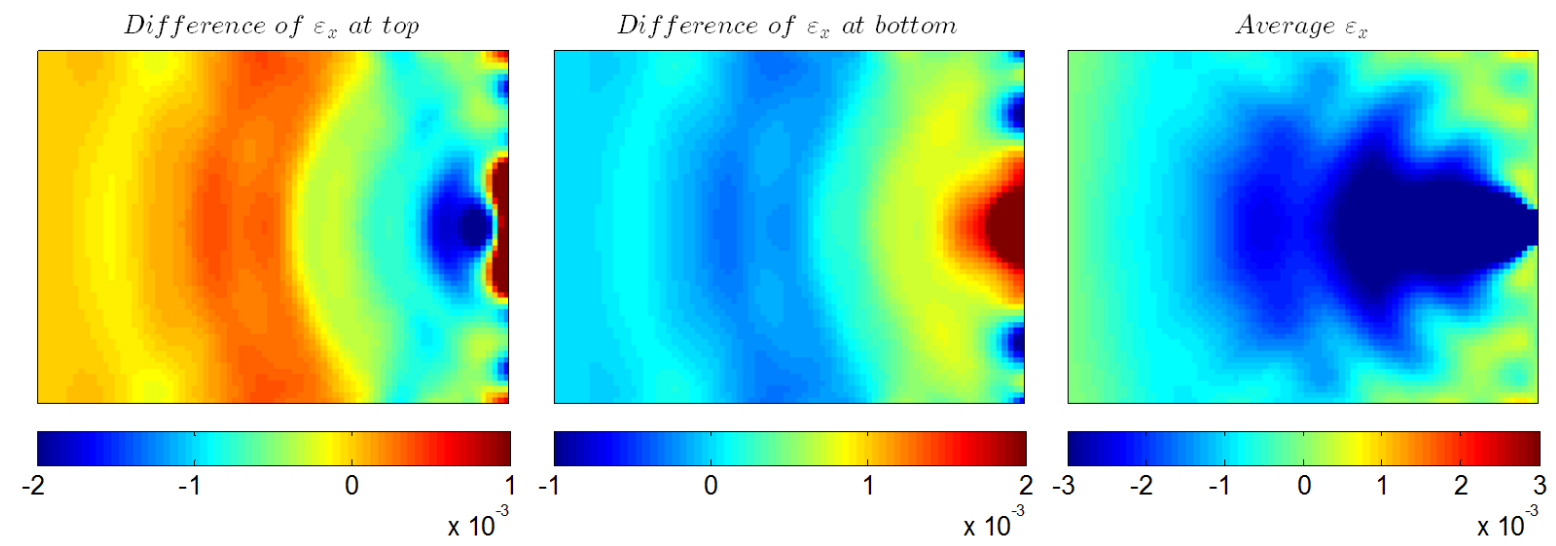

(a)
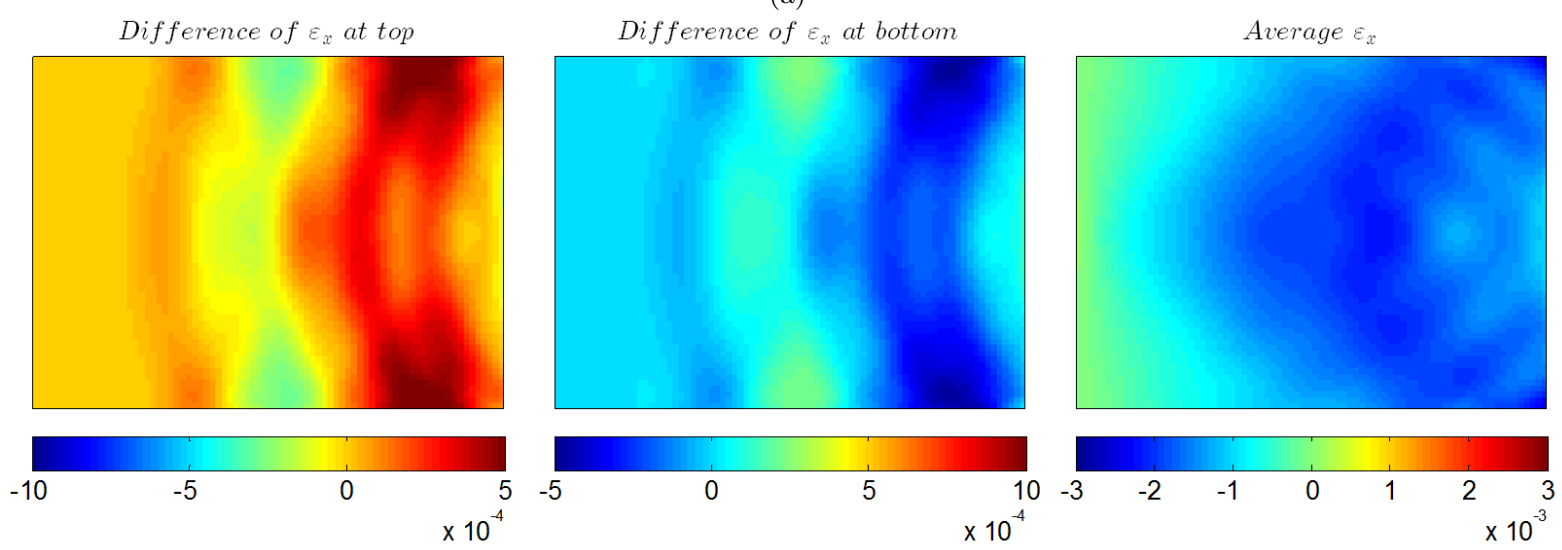

(b)
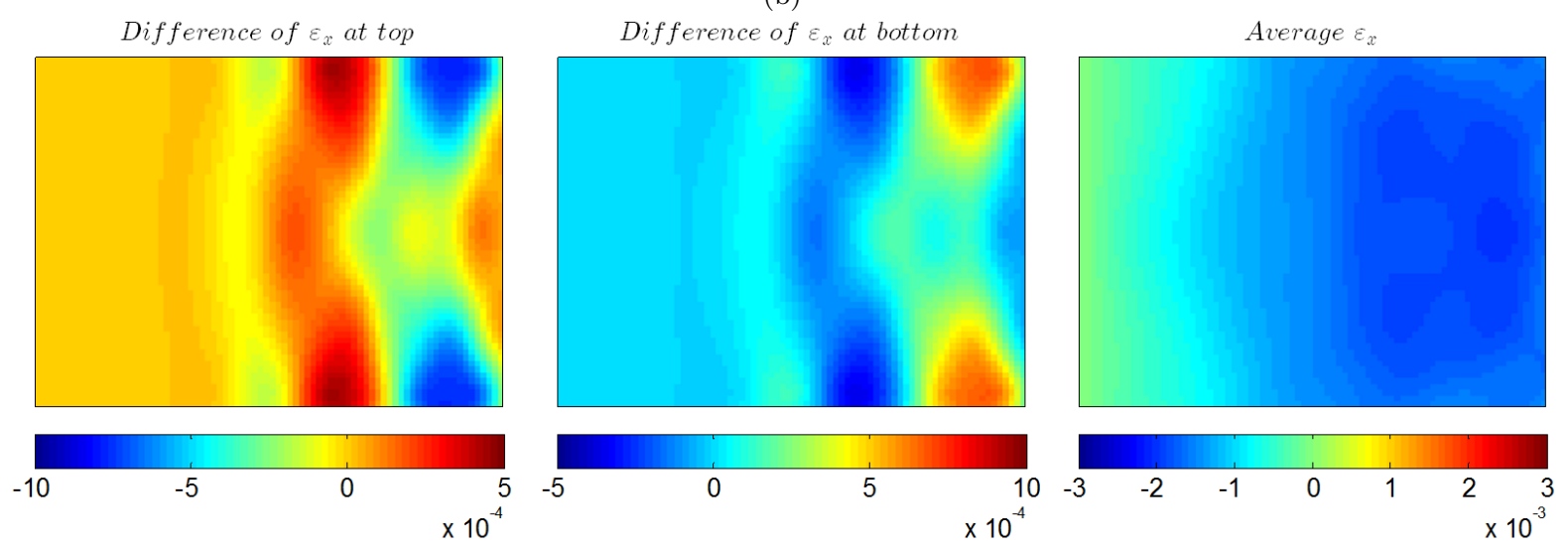

(c)

Figure 5: Differences in longitudinal strain at the top and bottom surfaces with tab. (a) $L=40 \mathrm{~mm}$, contact model (2) at 14 microseconds. (b) $L=60 \mathrm{~mm}$, contact model (2) at 19 microseconds. (c) $L=80 \mathrm{~mm}$, contact model (3) at 23 microseconds. 


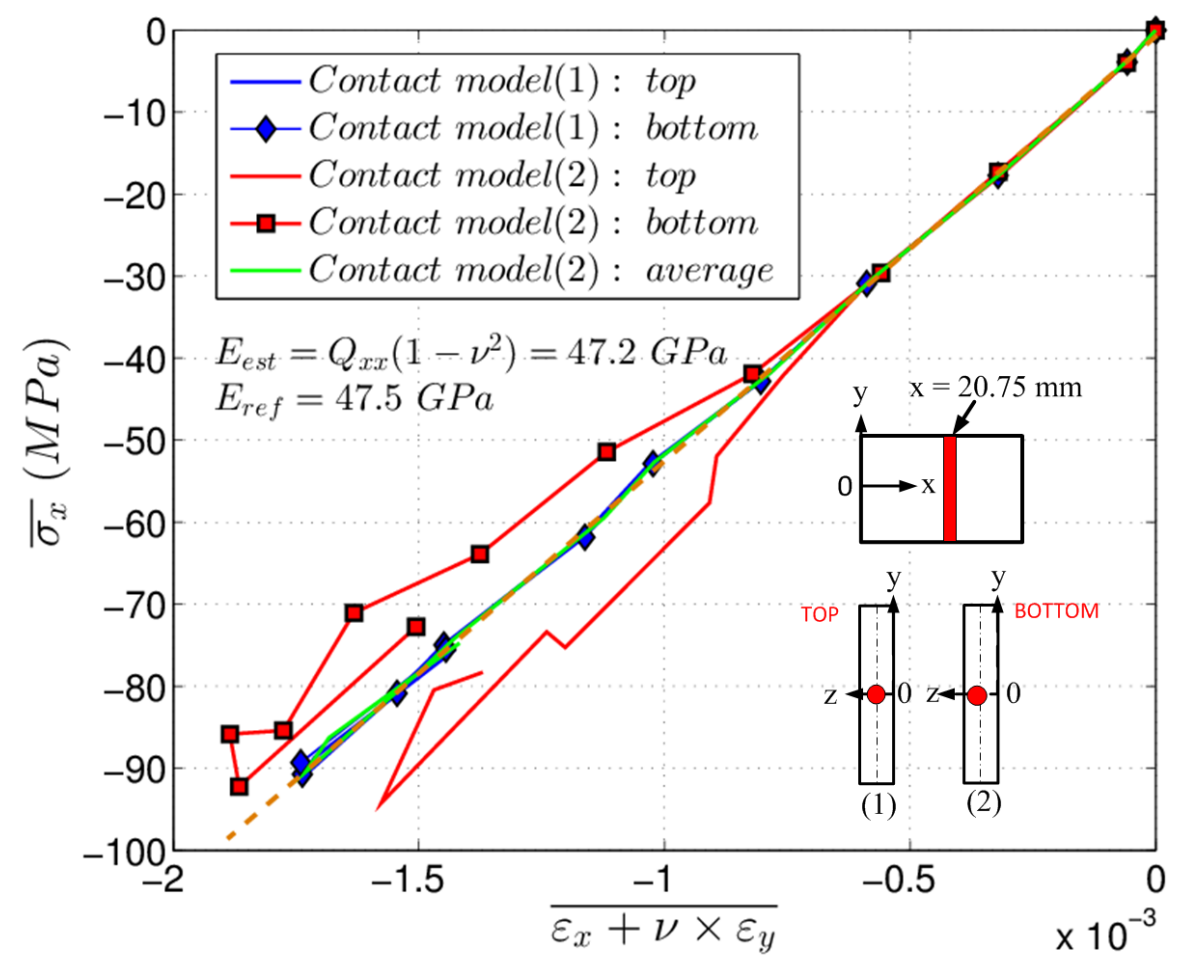

Figure 6: Stress-strain curves during the loading stage for contact models (1) and (2) at $x=20.75 \mathrm{~mm}$. $L=40 \mathrm{~mm}, \nu=0.3$. 


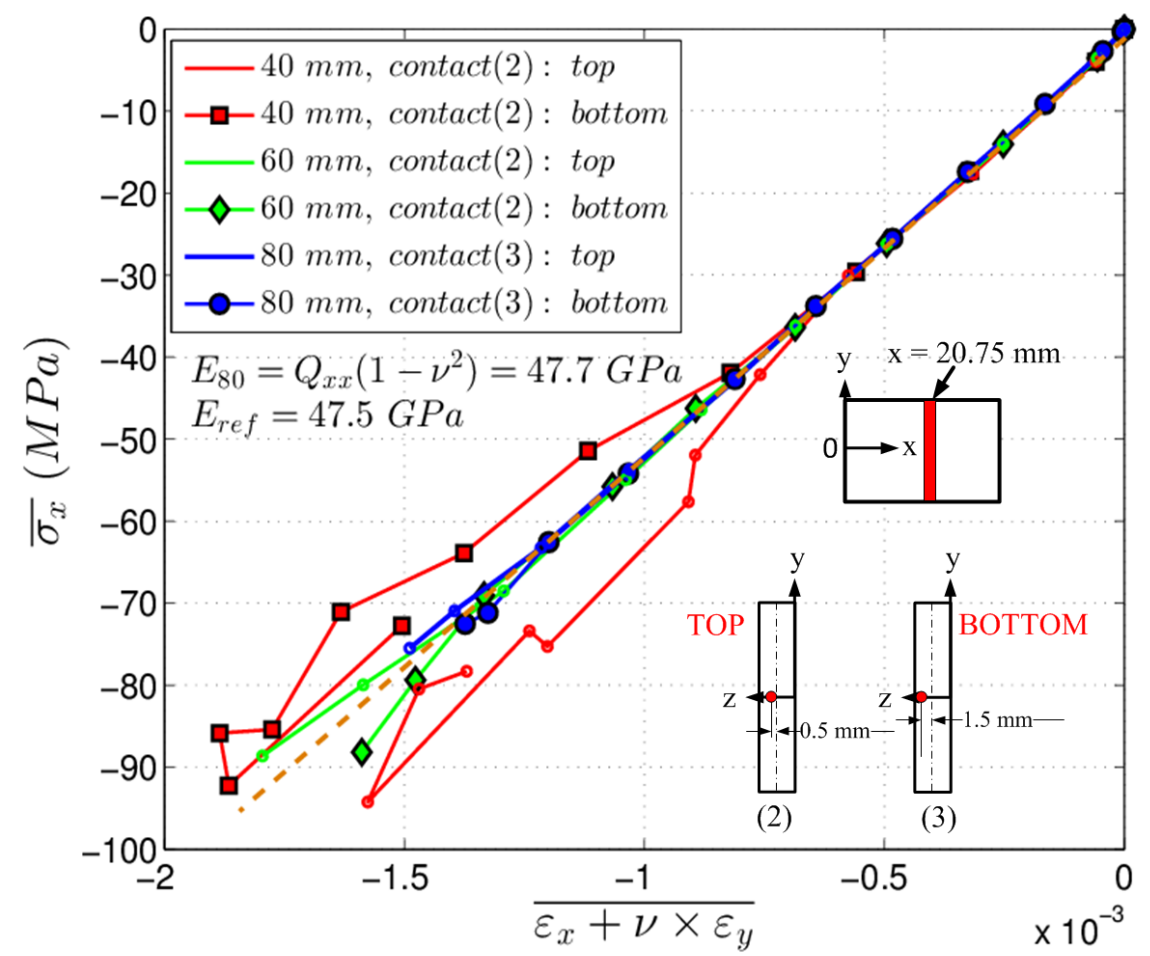

Figure 7: Stress-strain curves during the loading stage for offset contact models with different specimen lengths at $x=20.75 \mathrm{~mm}, \nu=0.3$. 


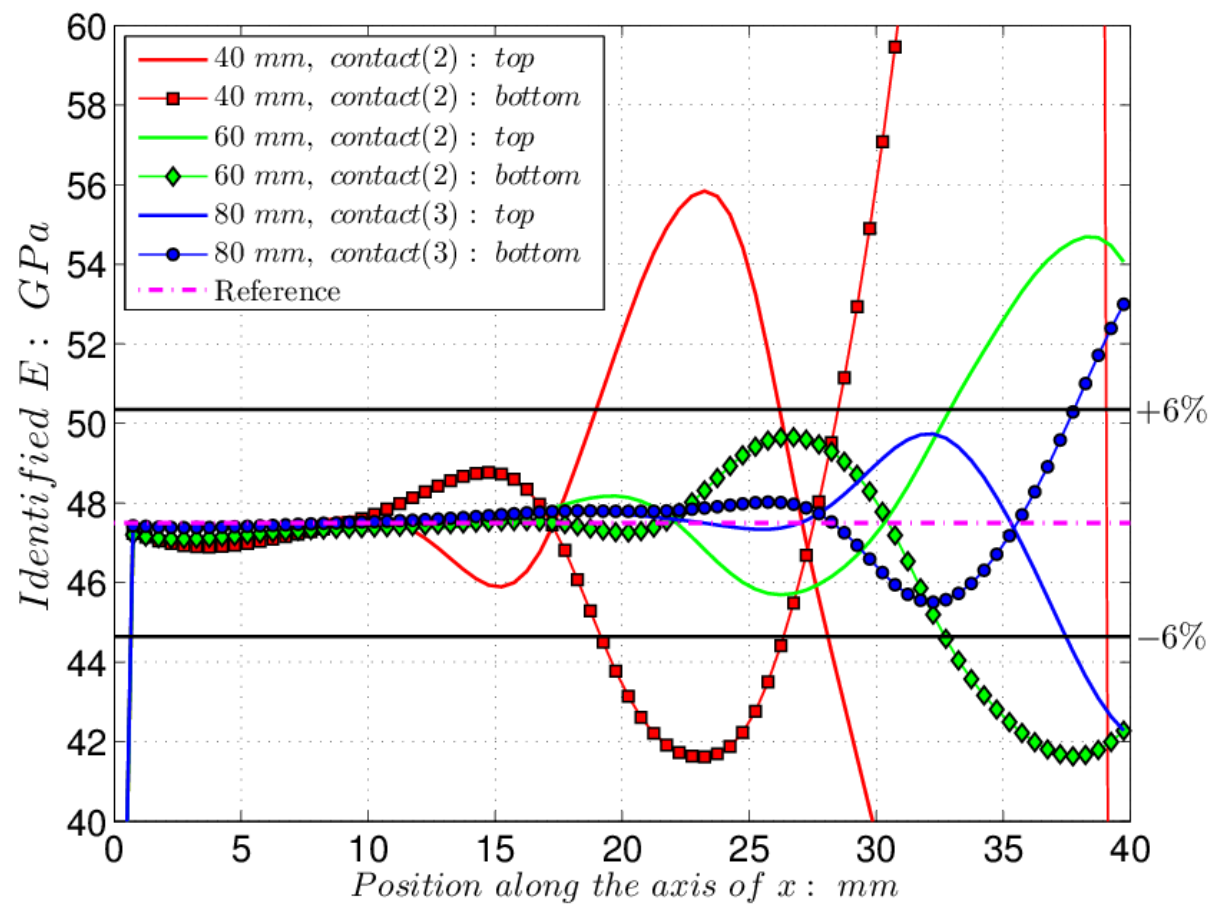

Figure 8: Identified Young's modulus from the stress-strain curves of specimens with different lengths with offset contact. 


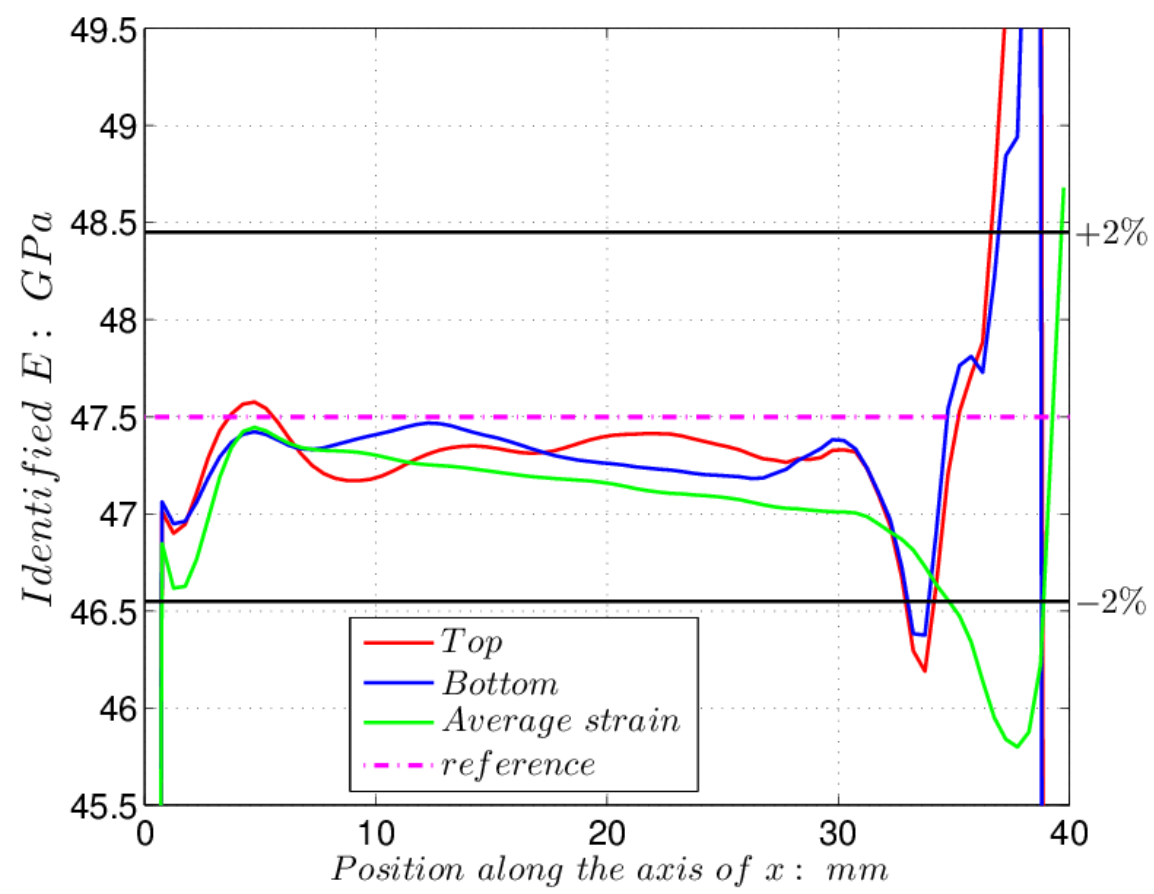

(a)

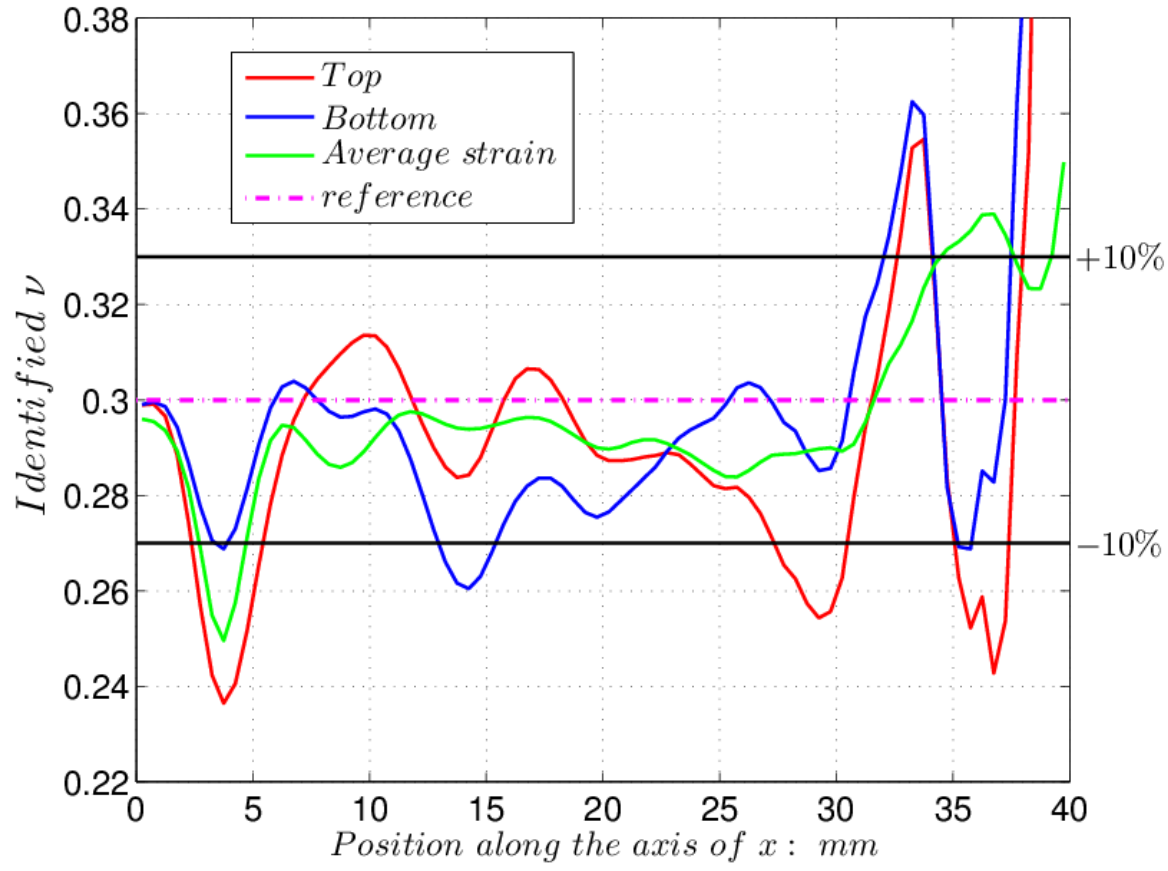

(b)

Figure 9: Identification of Young's modulus and Poisson's ratio from the over-determined system for the good contact model. $L=40 \mathrm{~mm}$. (a) Young's modulus. (b) Poisson's ratio. 


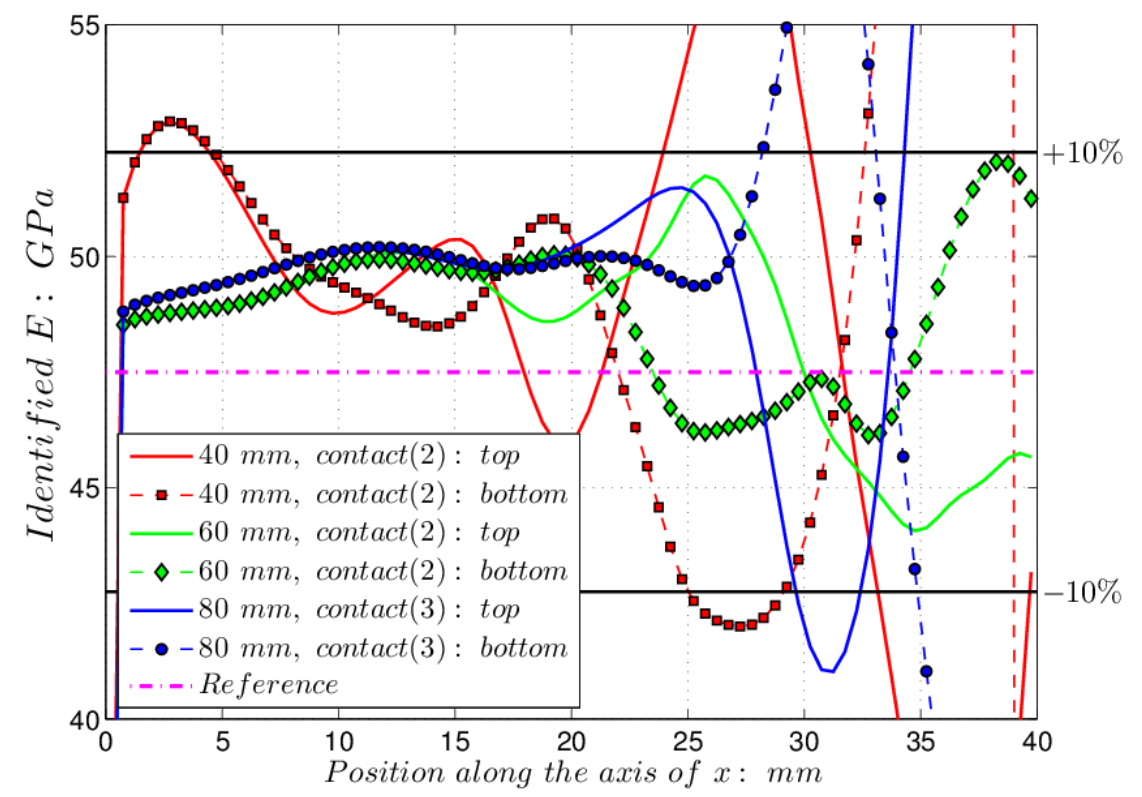

(a)

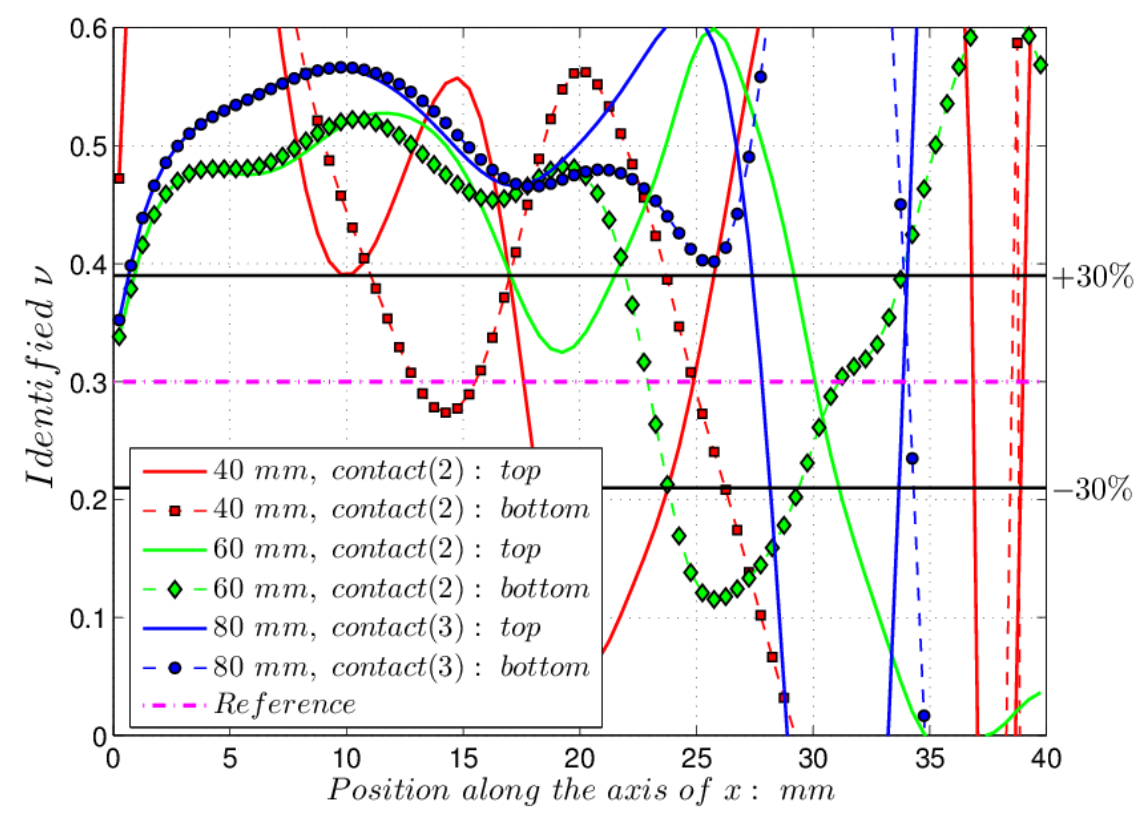

(b)

Figure 10: Identification of Young's modulus and Poisson's ratio from the over-determined system for the offset contact models. (a) Young's modulus. (b) Poisson's ratio. 


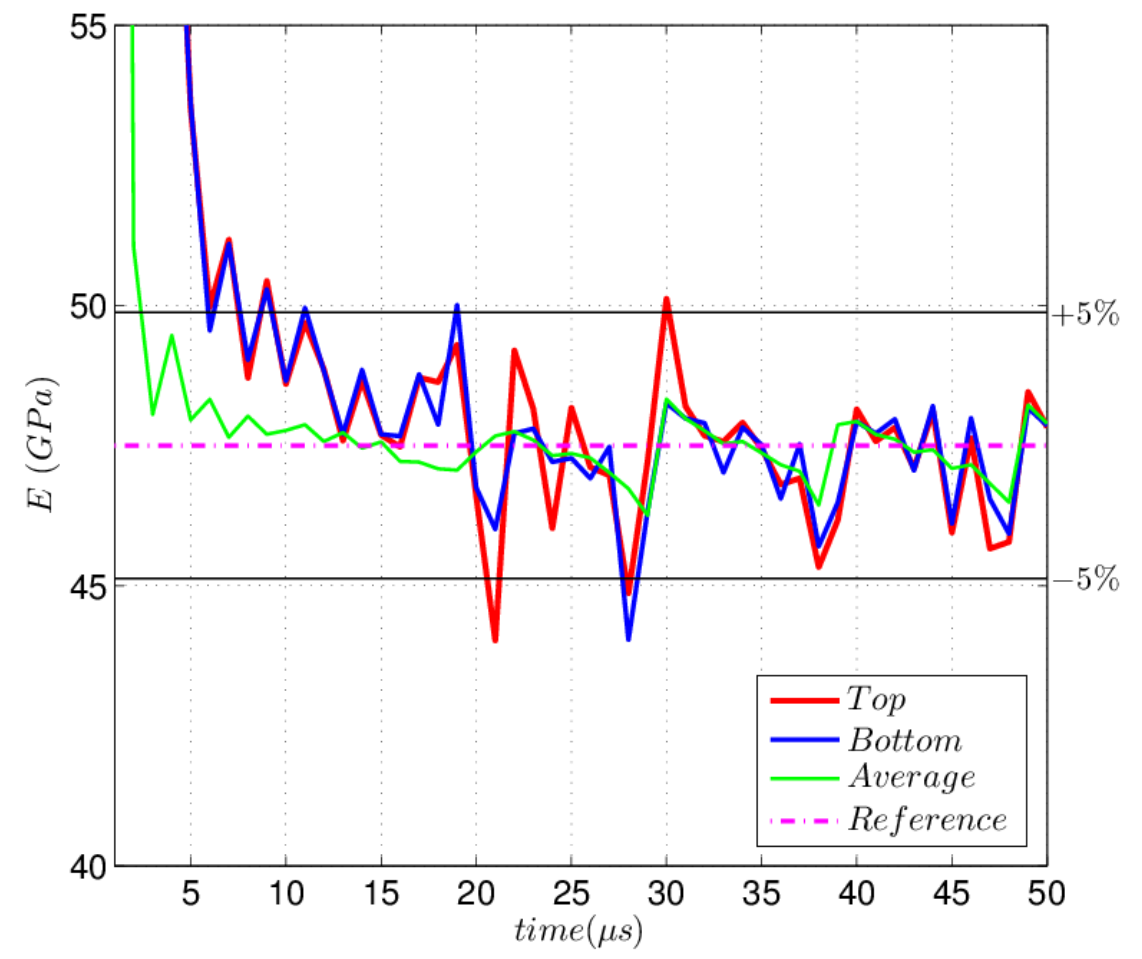

(a)

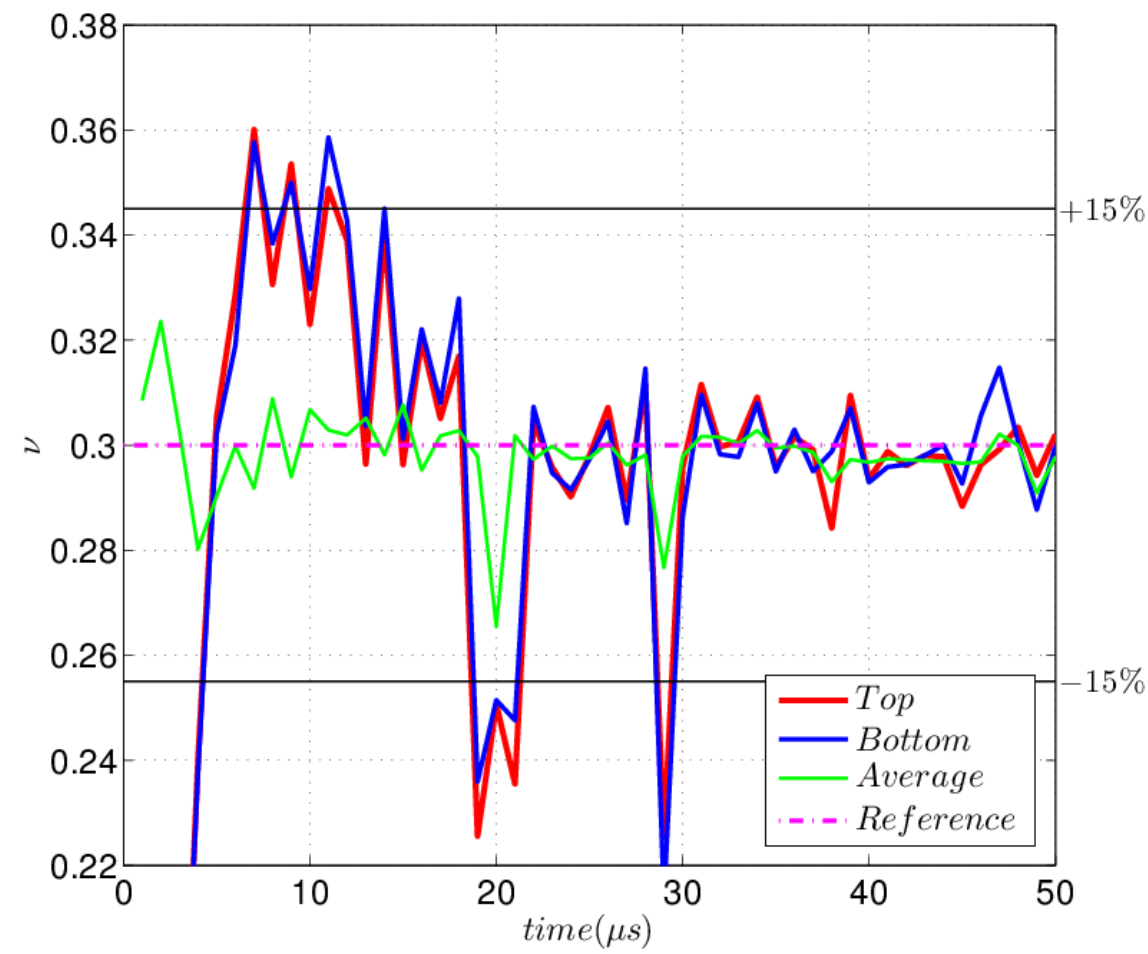

(b)

Figure 11: Identification of Young's modulus and Poisson's ratio from the whole field of view with the VFM. Data points: 80 by 60. Virtual mesh: 4 by 3. $L=40 \mathrm{~mm}$. Contact Model (1). (a) Young's modulus. (b) Poisson's ratio. 


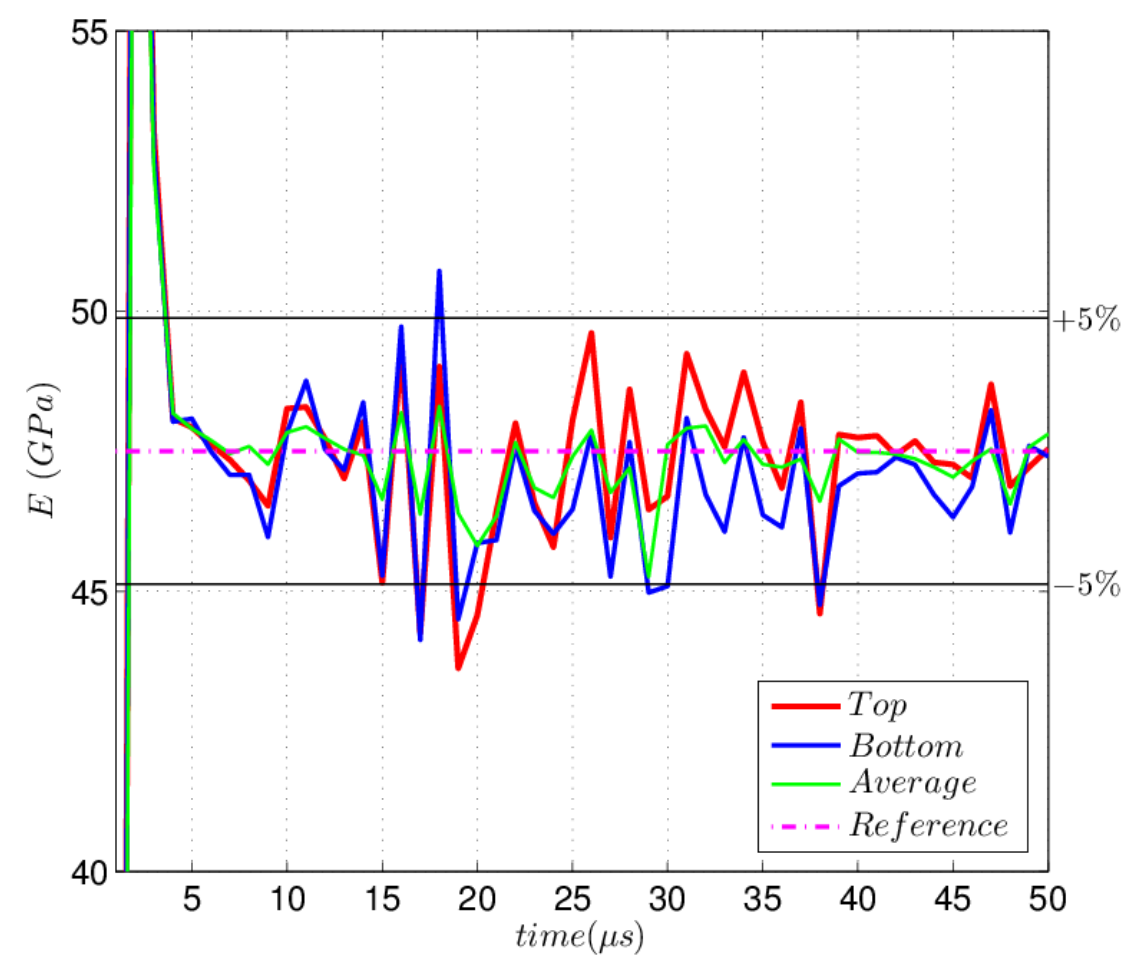

(a)

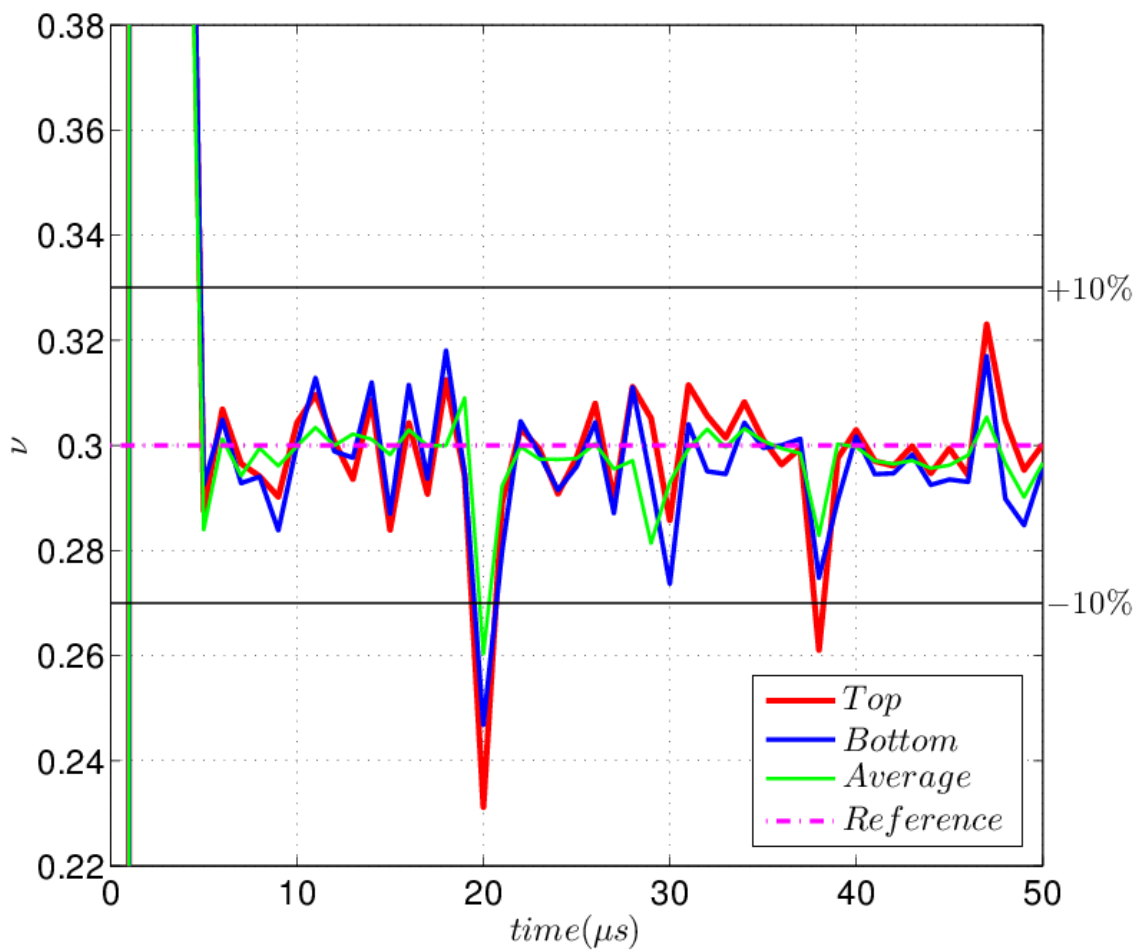

(b)

Figure 12: Identification of Young's modulus and Poisson's ratio from the central area with the VFM. Data points: 64 by 60 . Virtual mesh: 4 by $3 . L=40 \mathrm{~mm}$. Contact Model (1). (a) Young's modulus. (b) Poisson's ratio. 


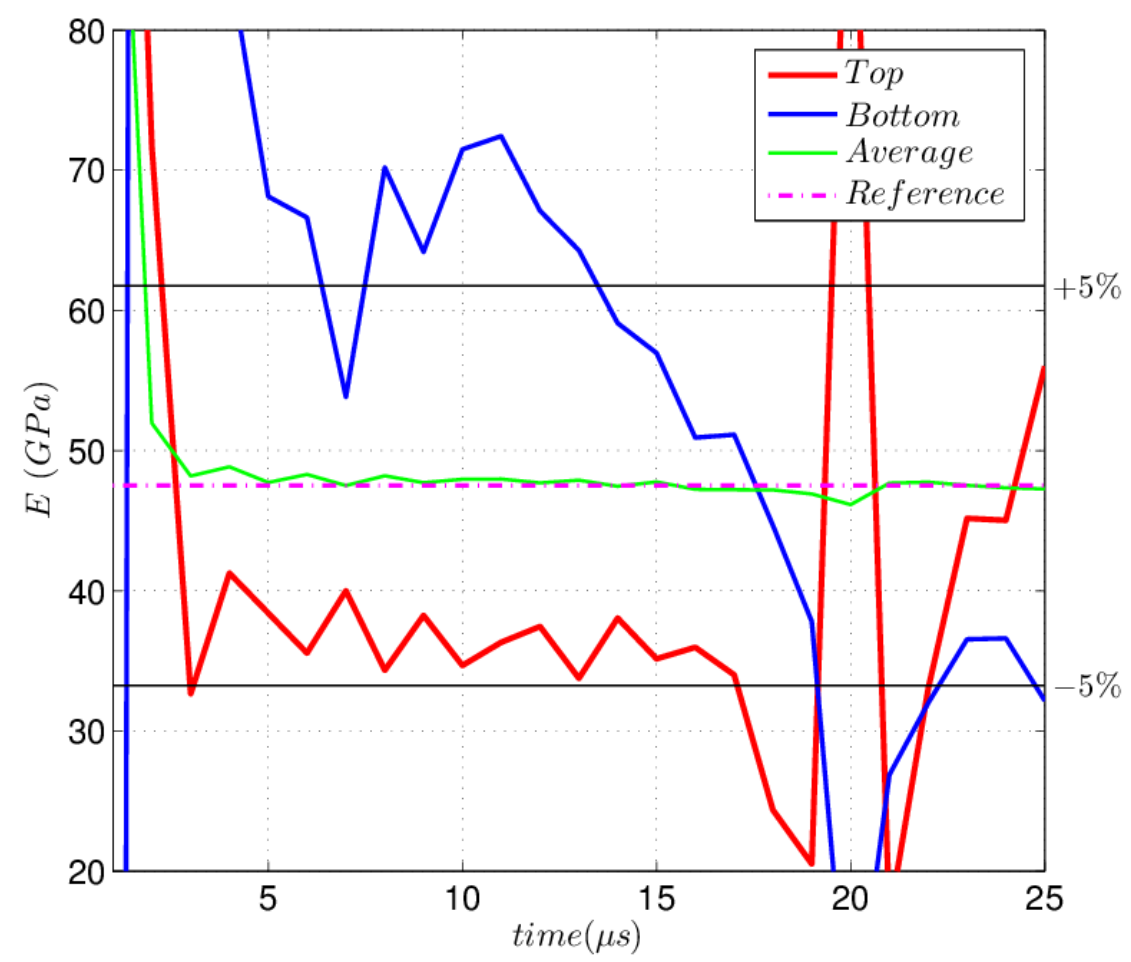

(a)

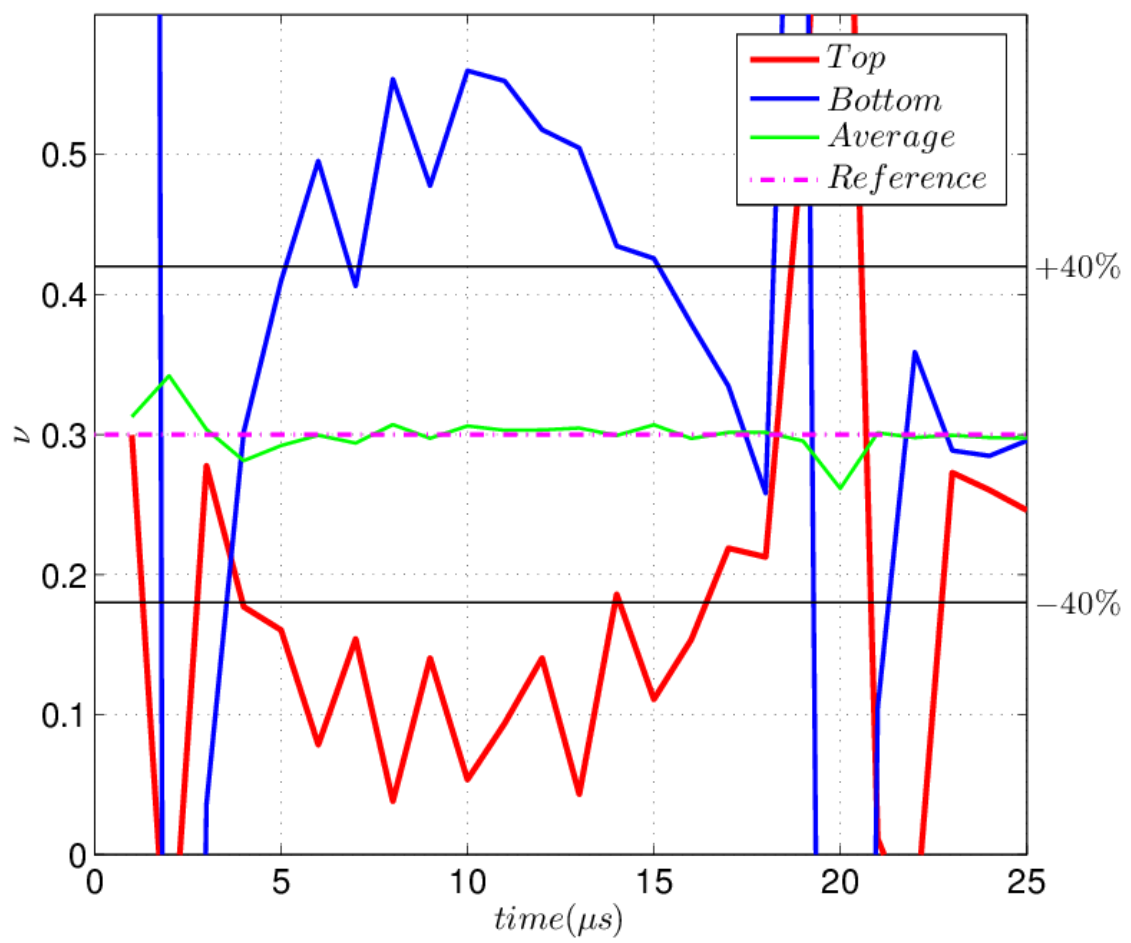

(b)

Figure 13: Identification of Young's modulus and Poisson's ratio with the VFM. Data points: 80 by 60 . Virtual mesh: 4 by 3. $L=40 \mathrm{~mm}$. Contact Model (2). (a) Young's modulus. (b) Poisson's ratio. 


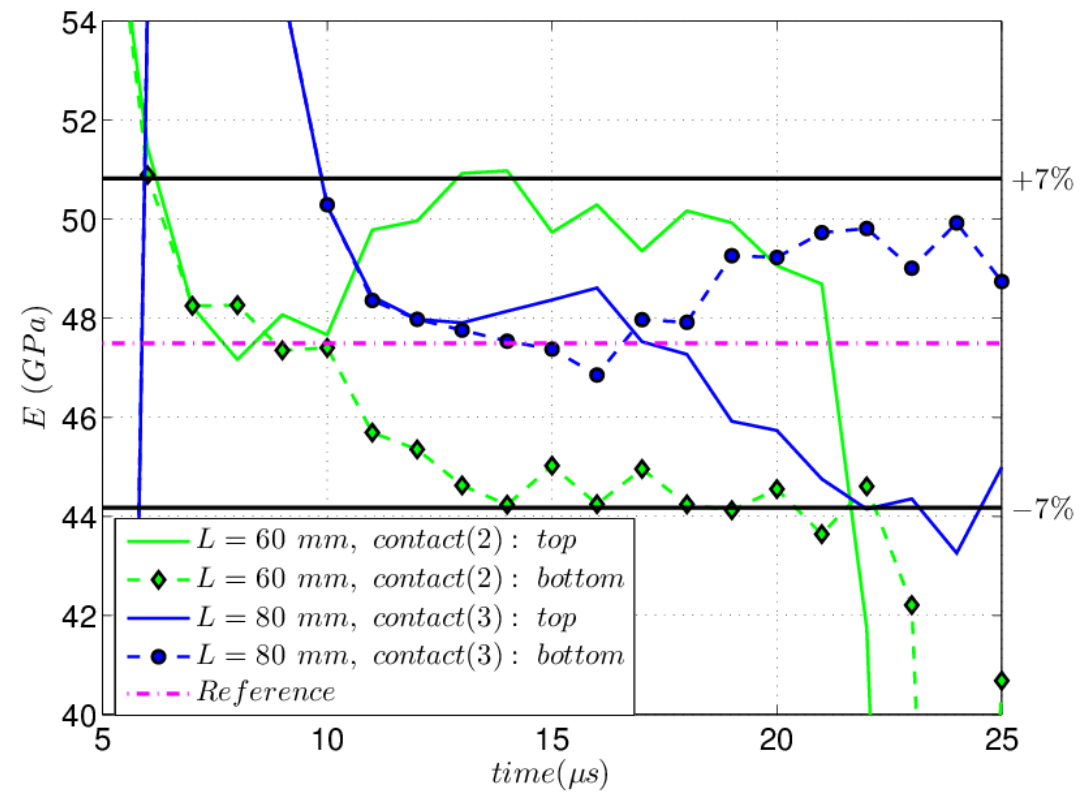

(a)

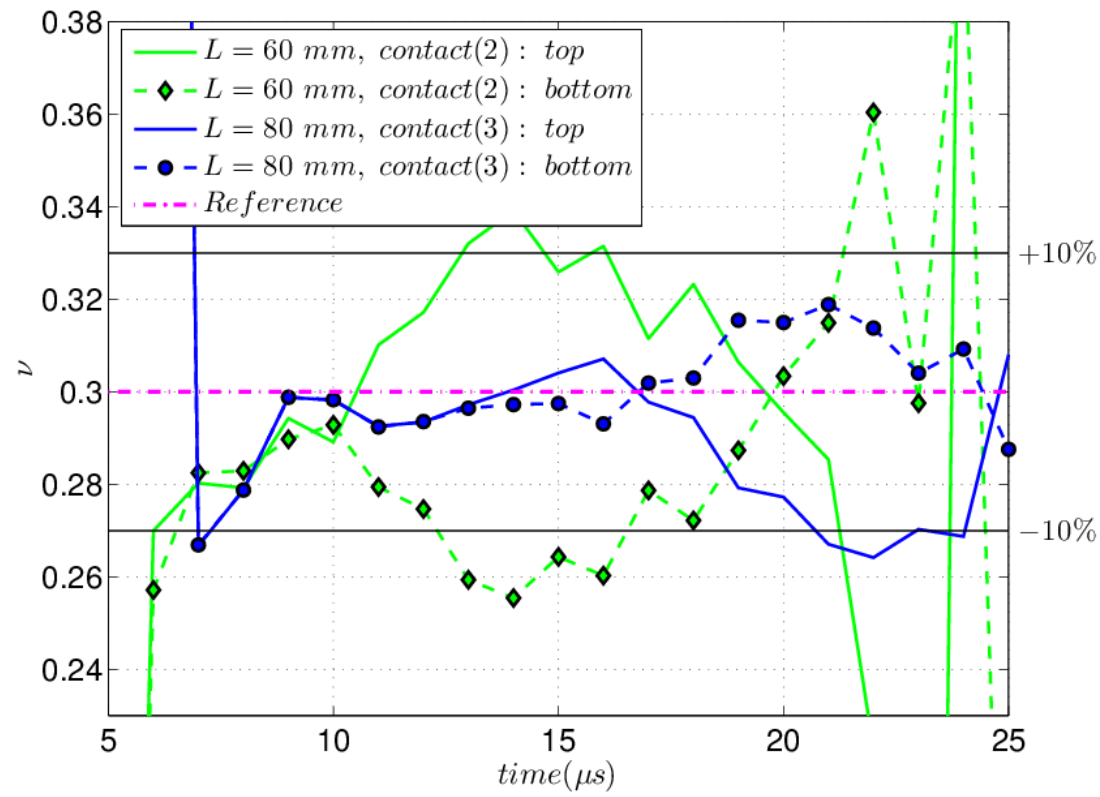

(b)

Figure 14: Identification of Young's modulus and Poisson's ratio with the VFM. Data points: 80 by 60. Virtual mesh: 4 by 3. Offset contact models \& longer specimens. (a) Young's modulus. (b) Poisson's ratio. 

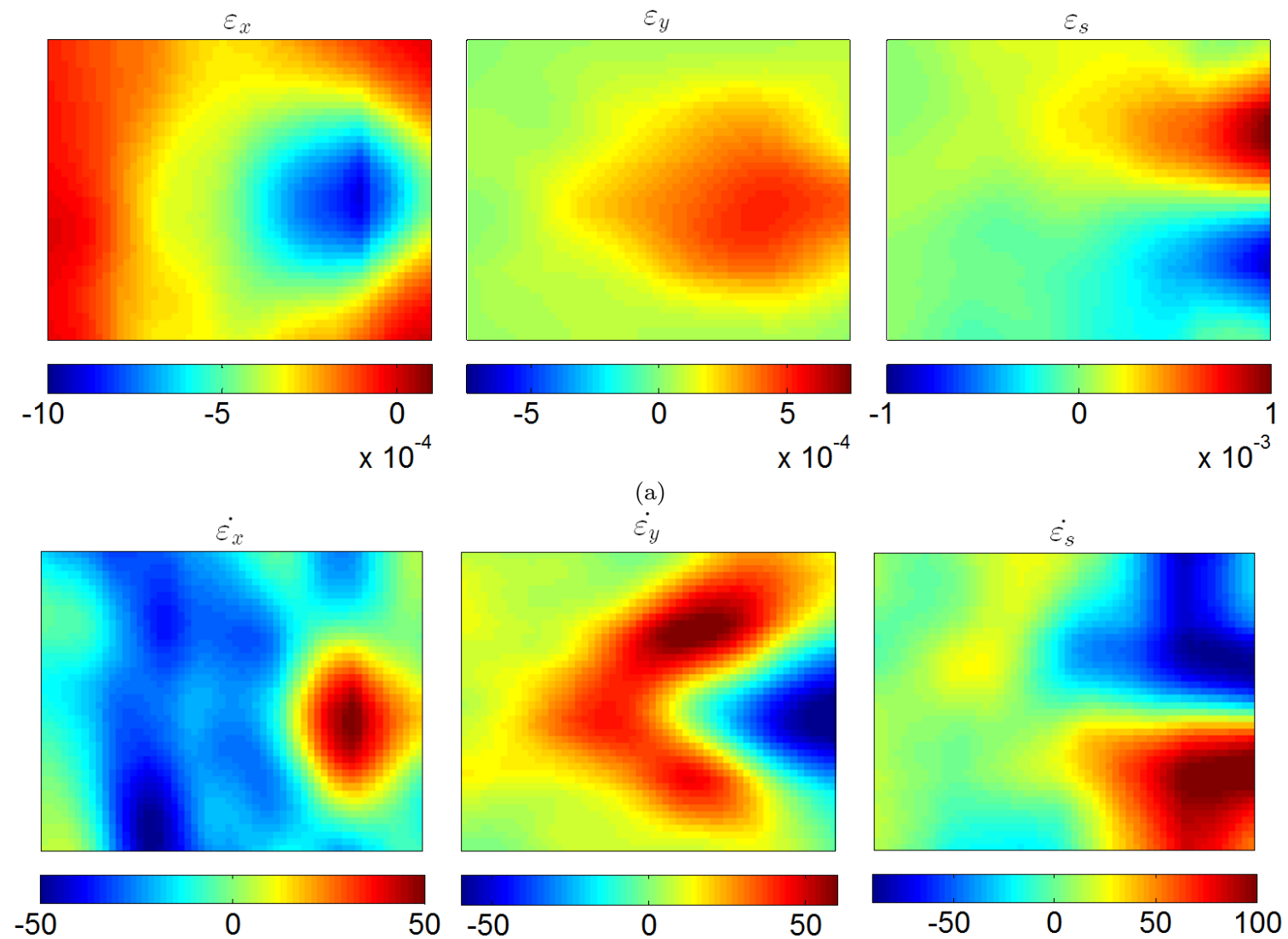

(b)
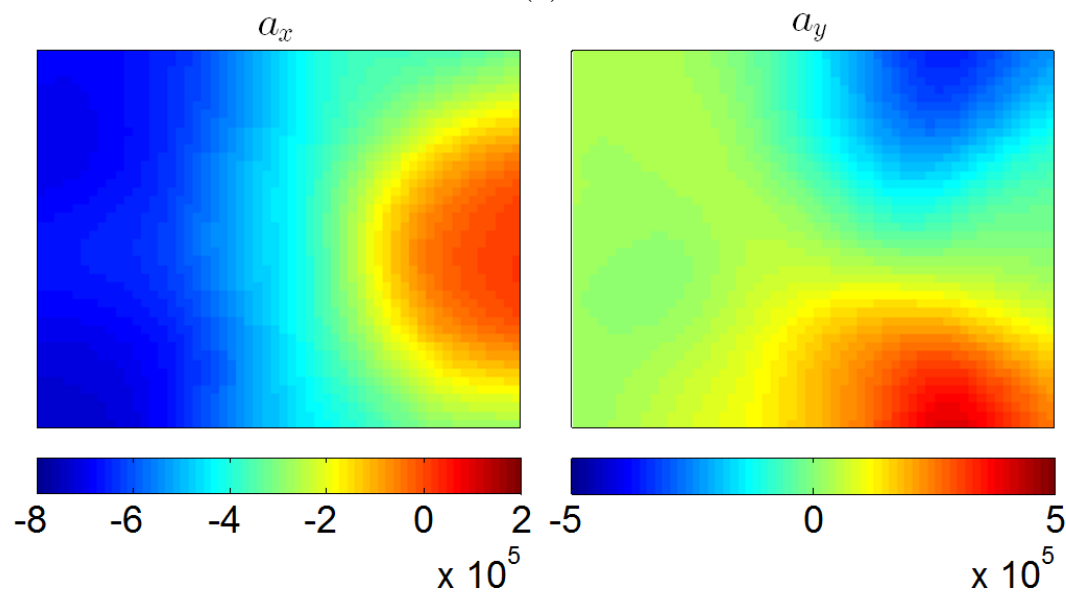

(c)

Figure 15: Strain and acceleration maps for specimen 1 at 9 microseconds. Data points: 56 by 44 . (a) Strain. (b) Strain rate (in $s^{-1}$ ). (c) Acceleration (in $m \cdot s^{-2}$ ). 

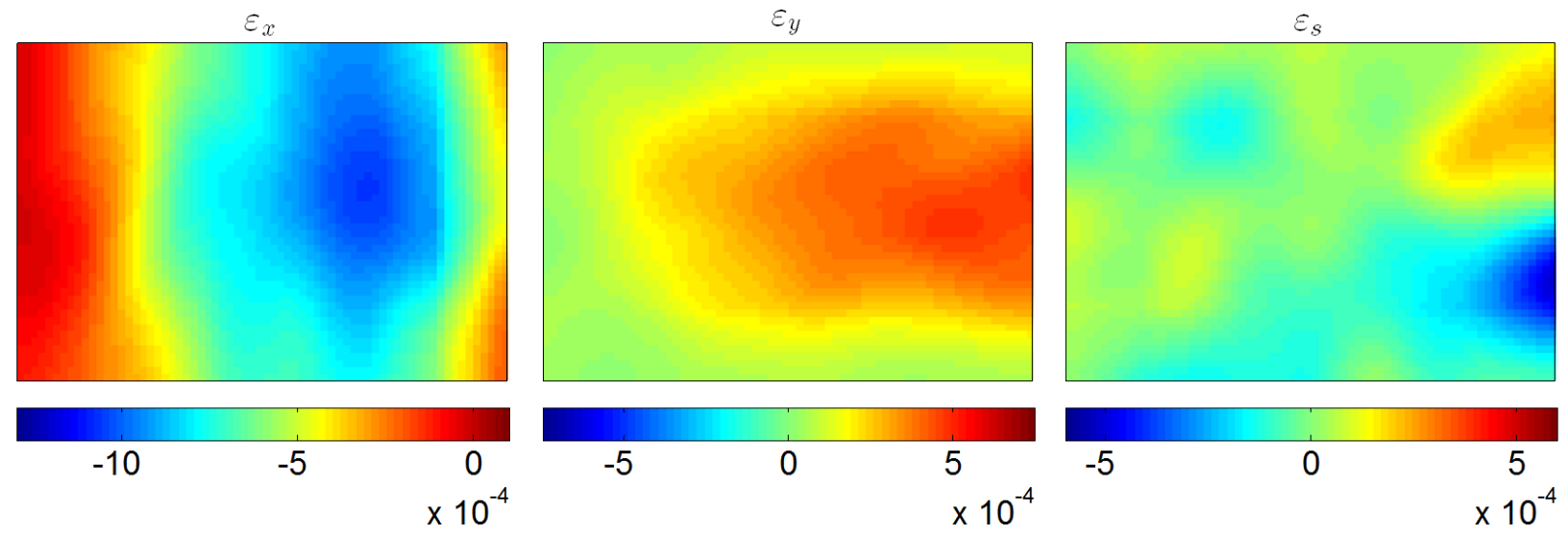

(a)
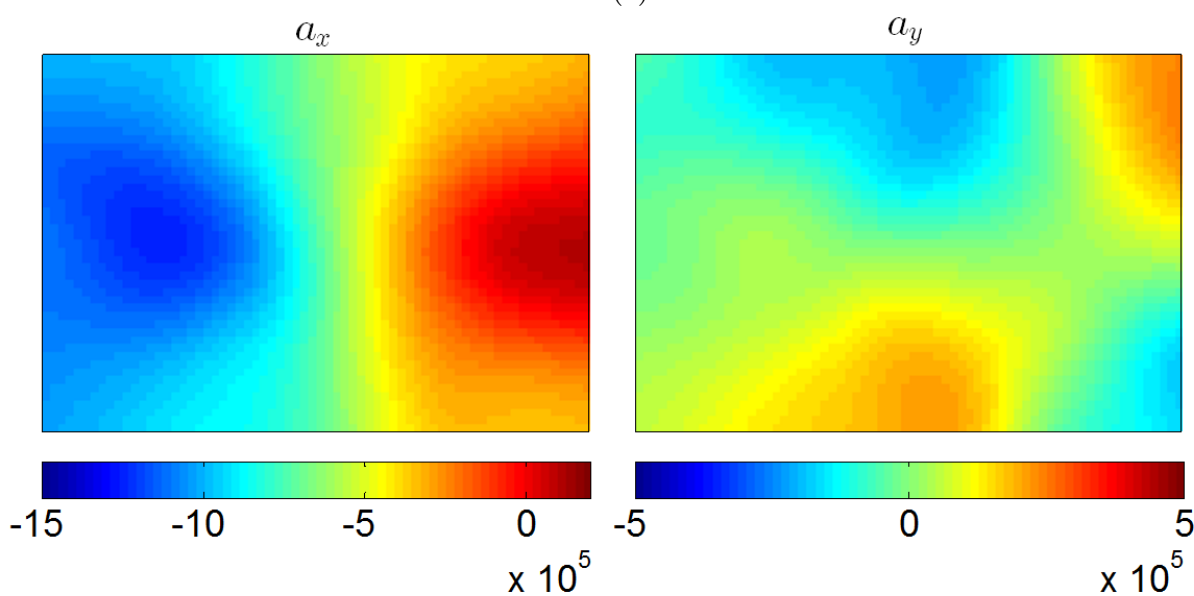

(b)

Figure 16: Strain and acceleration maps for specimen 2 at 13 microseconds. Data points: 68 by 47. (a) Strain. (b) Acceleration (in $m \cdot s^{-2}$ ). 

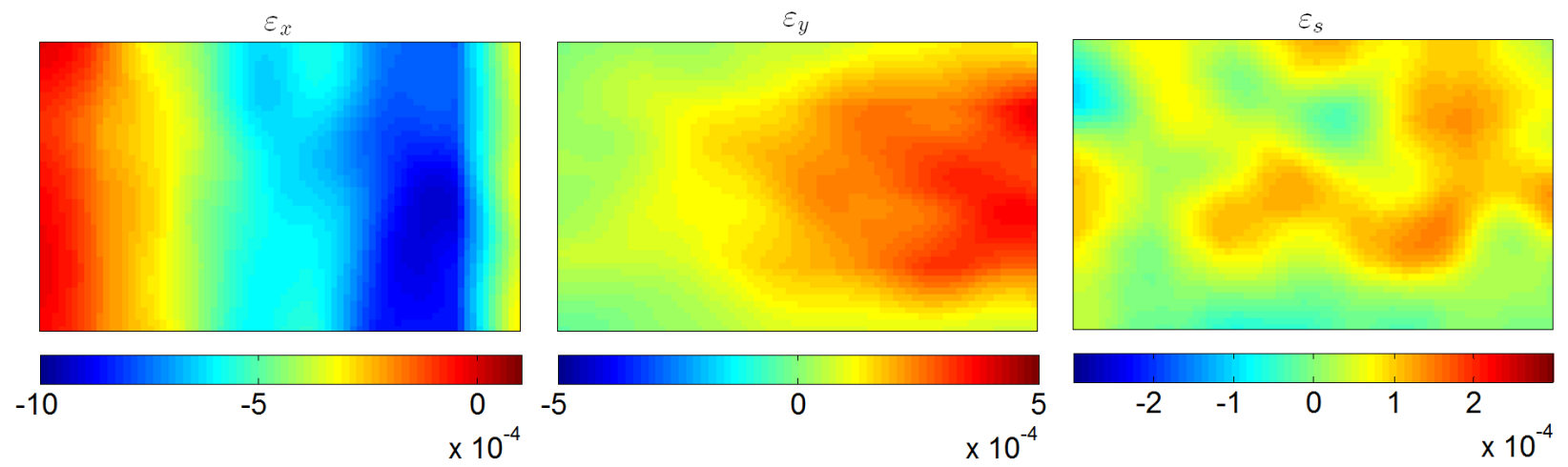

(a)
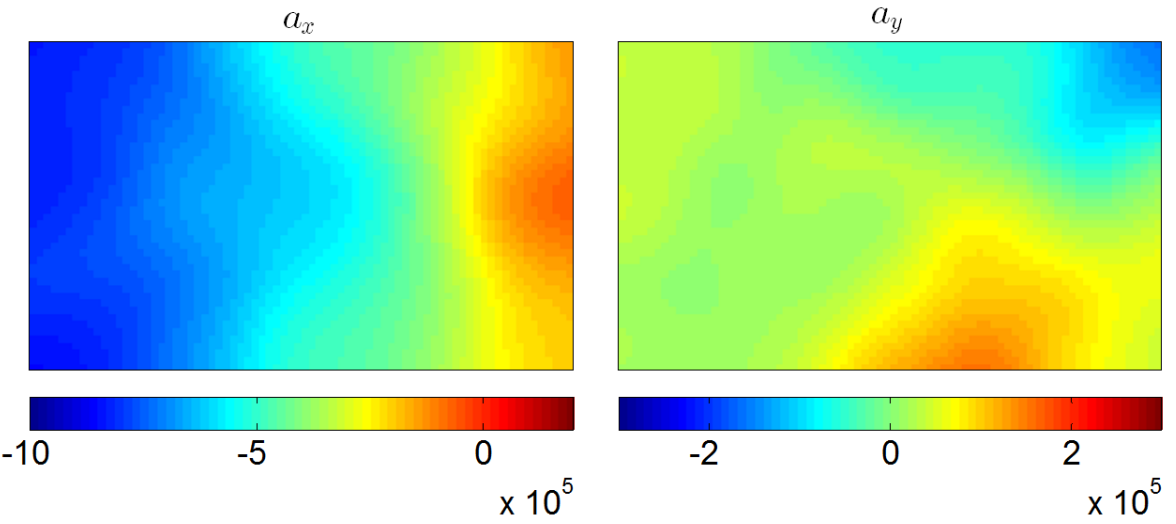

(b)

Figure 17: Strain and acceleration maps for specimen 3 at 17 microseconds. Data points: 76 by 46 . (a) Strain. (b) Acceleration (in $m \cdot s^{-2}$ ). 


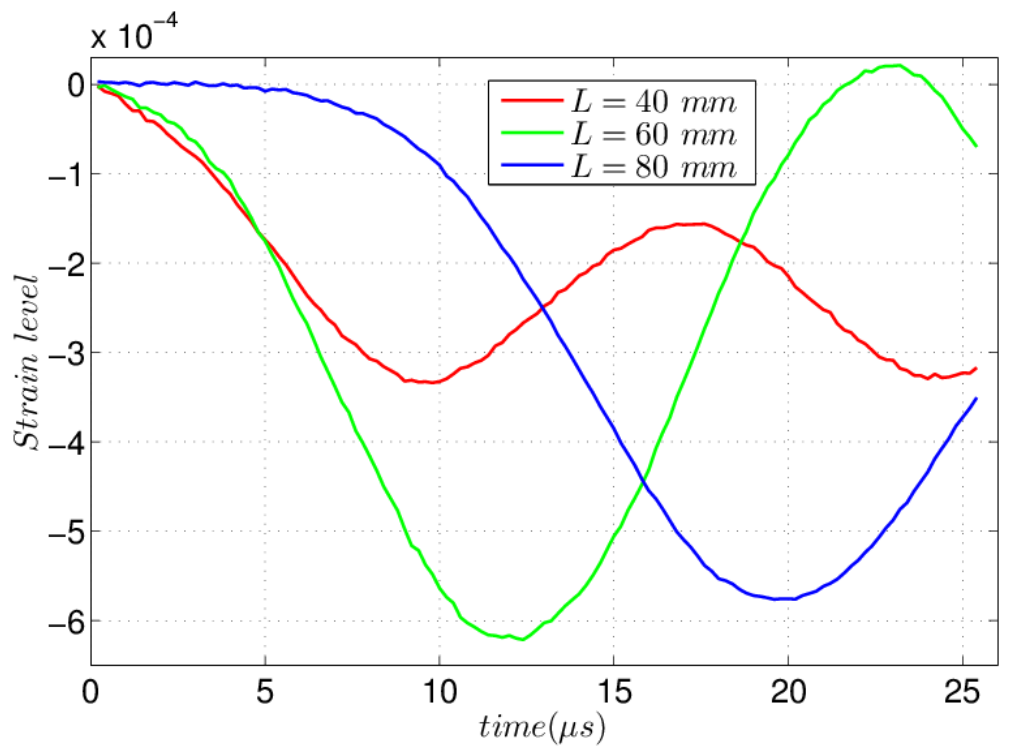

(a)

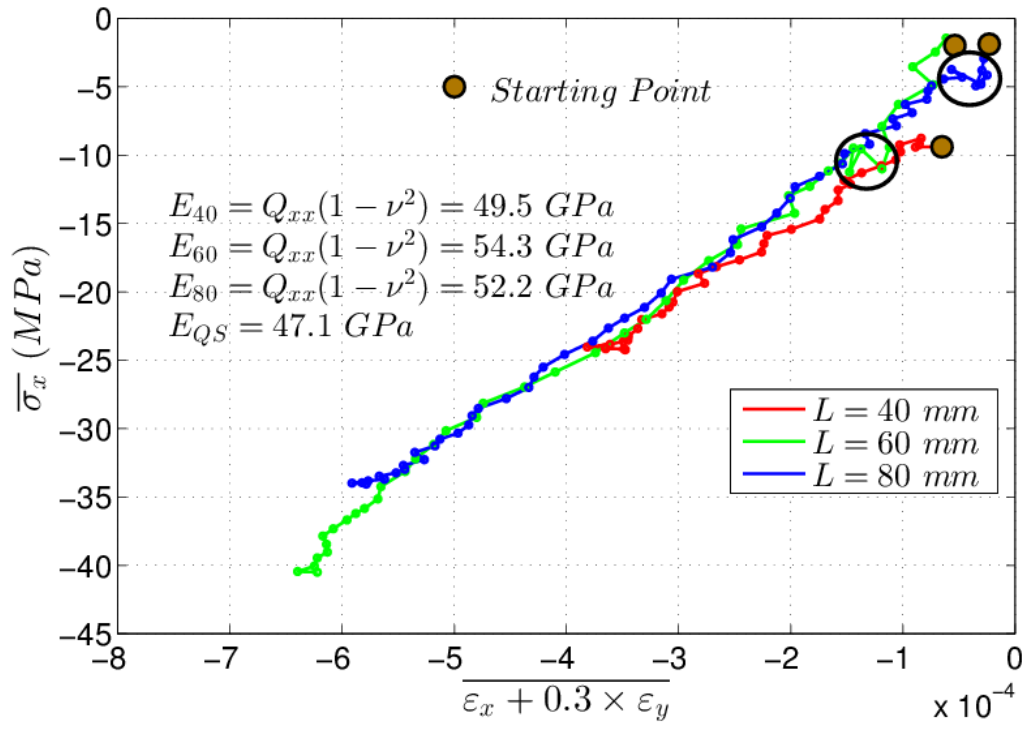

(b)

Figure 18: Strain levels and stress-strain curves for three specimens. (a) Longitudinal strain levels for the three tests. (b) Stress-strain curves for the three specimens at $x=17.7 \mathrm{~mm} . \nu=0.3$. 


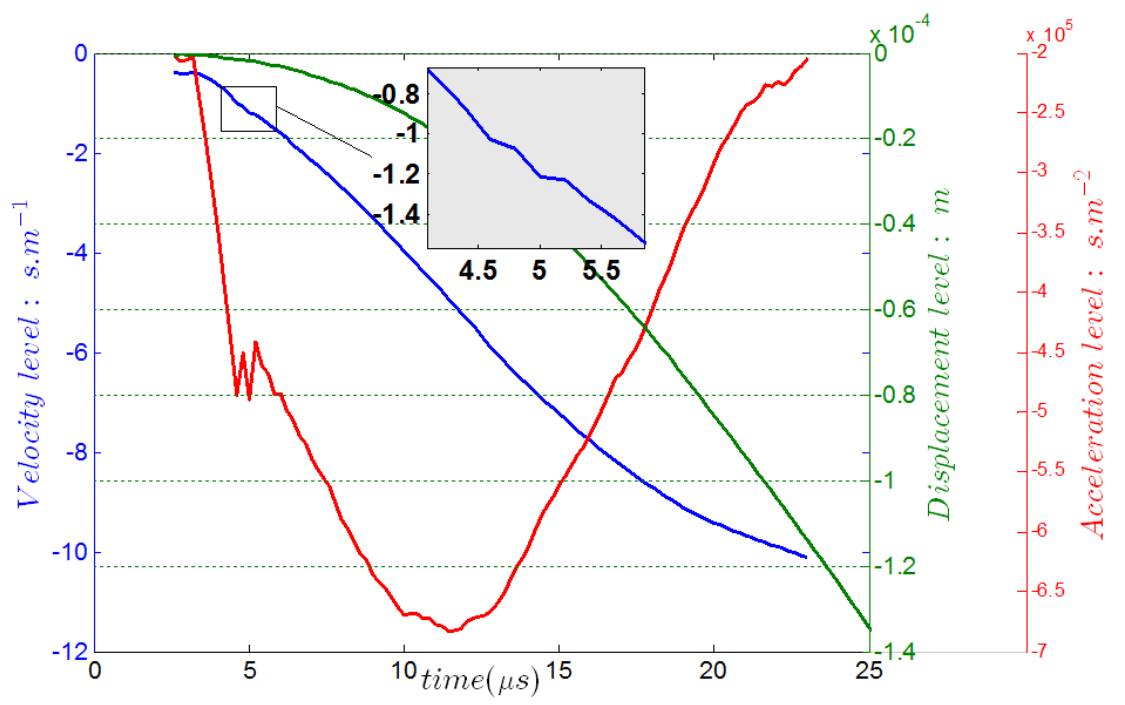

(a)

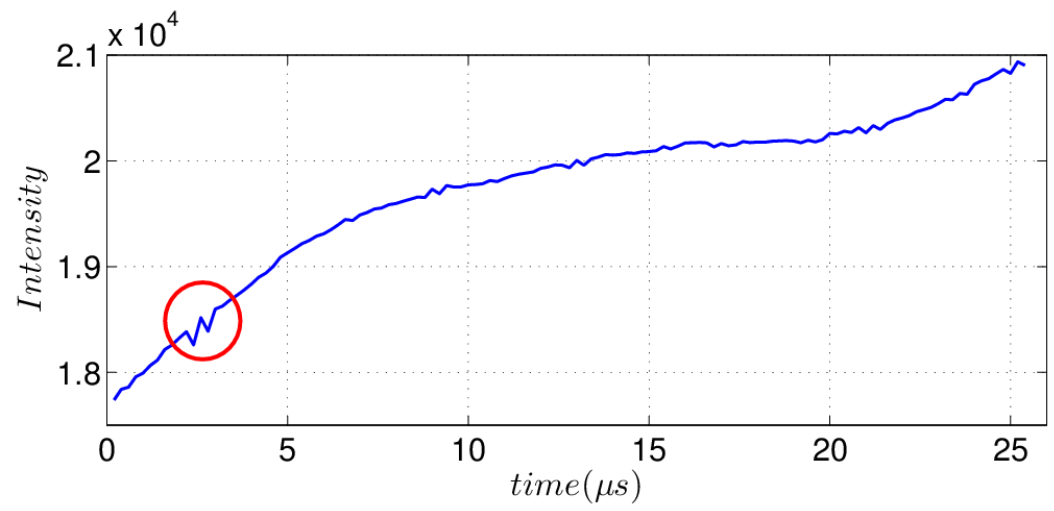

(b)

Figure 19: Profiles of displacement, velocity, acceleration and intensity for specimen 2. (a) Longitudinal displacement, velocity and acceleration. (b) Average grey level intensity of the raw images. 


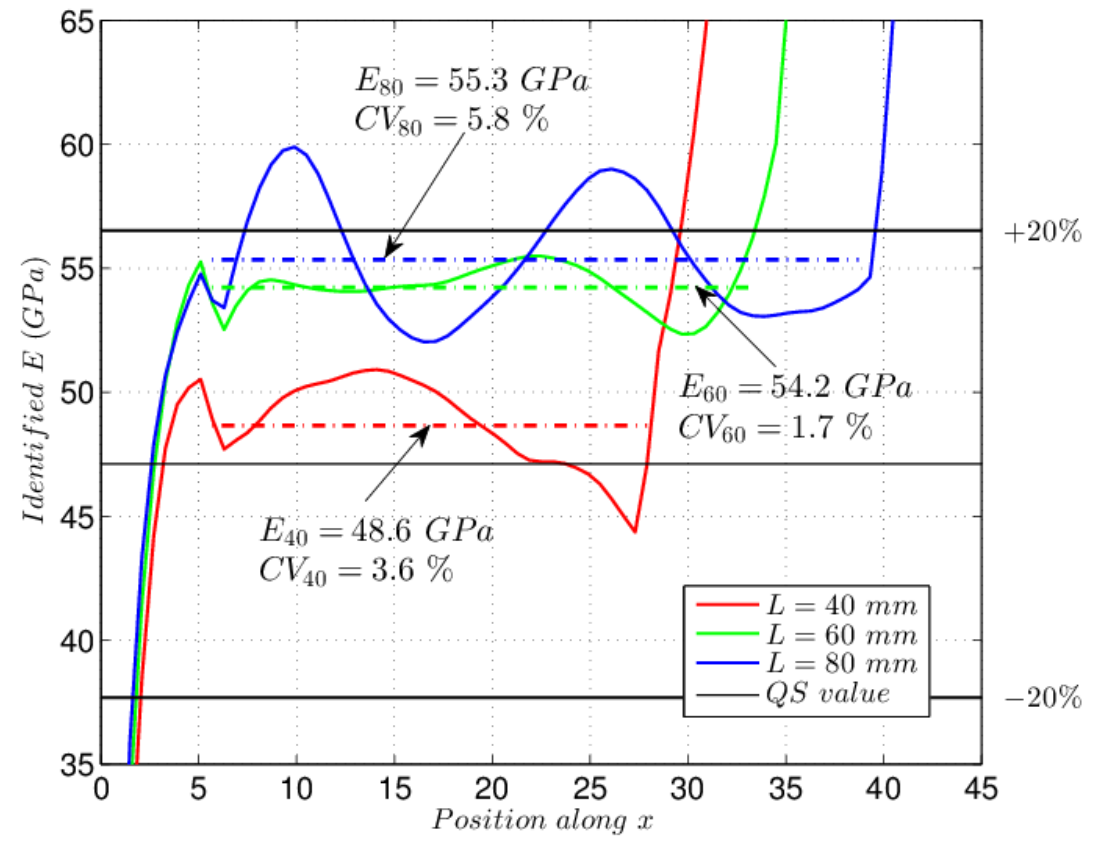

(a)

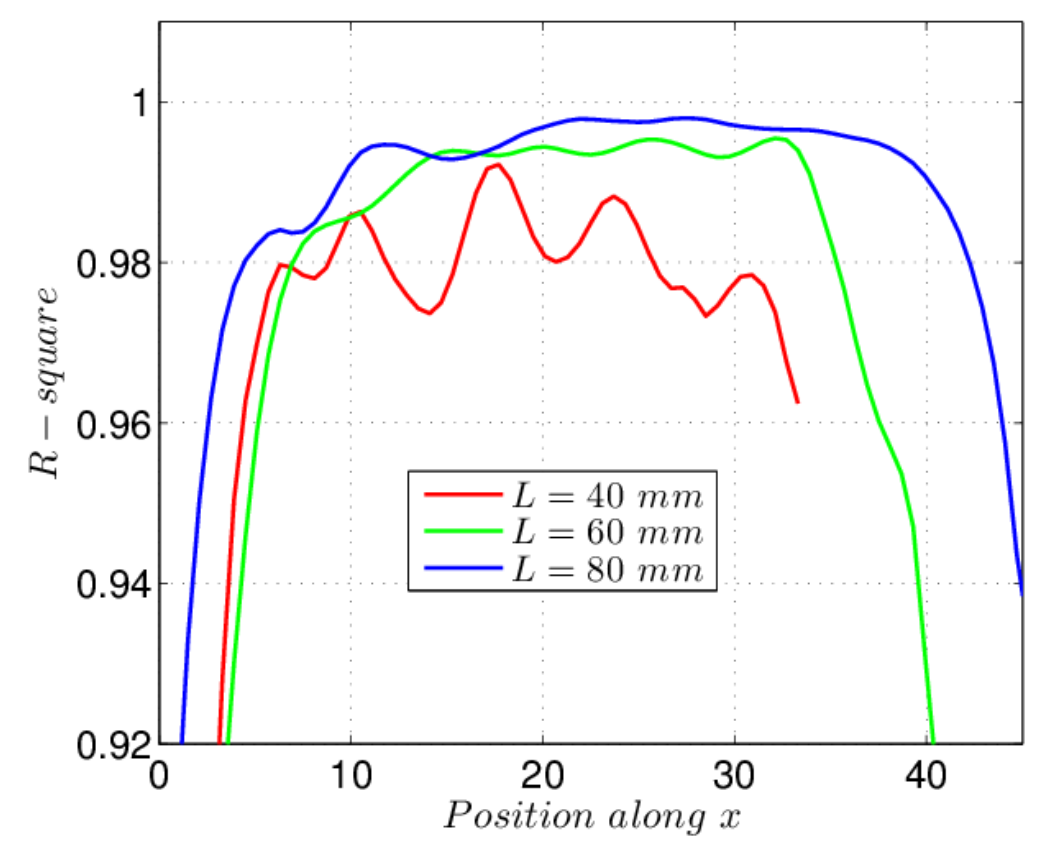

(b)

Figure 20: Identification of Young's modulus for three specimens. (a) Identified Young's modulus. (b) Correlation coefficients. 


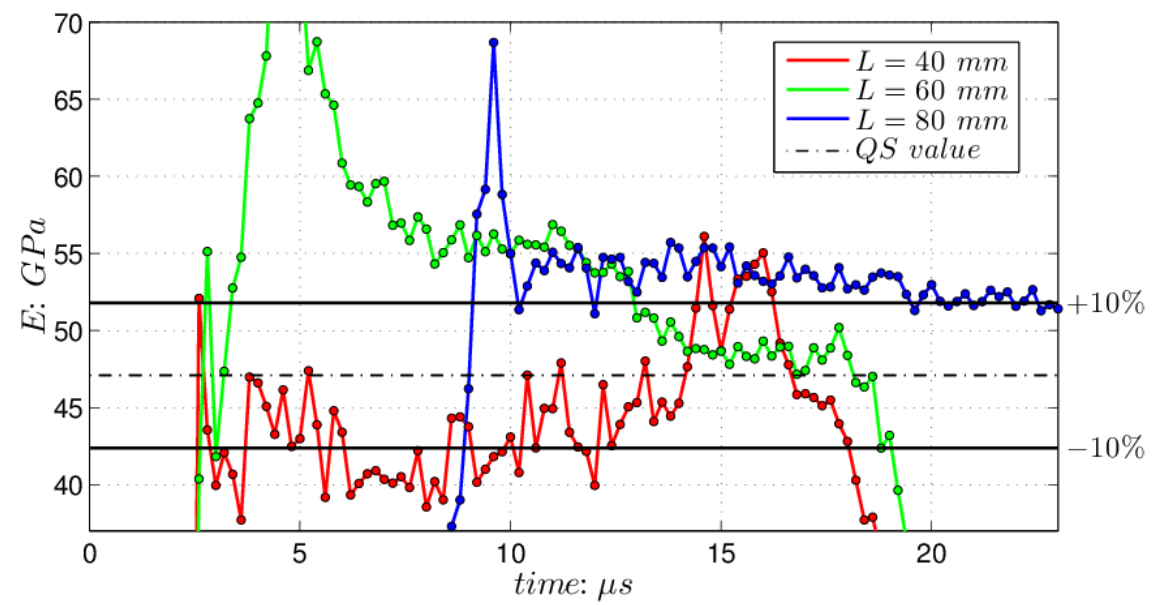

(a)

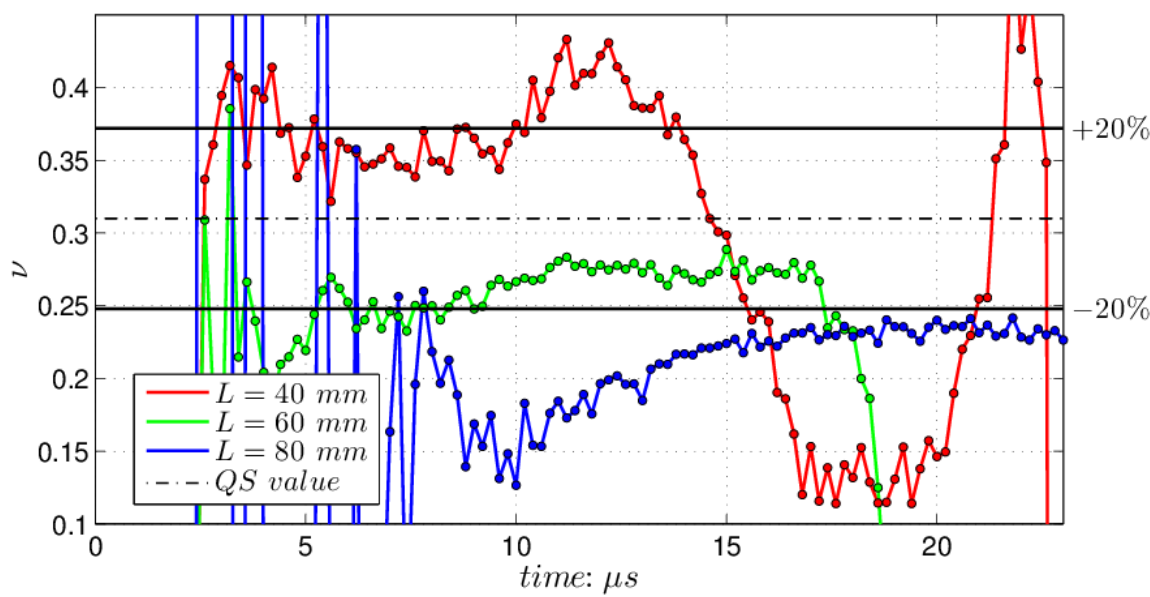

(b)

Figure 21: Identified Young's modulus and Poisson's ratios with the VFM for the three specimens. Virtual mesh: $m=13, n=3$. (a) Young's modulus. (b) Poisson's ratio. 


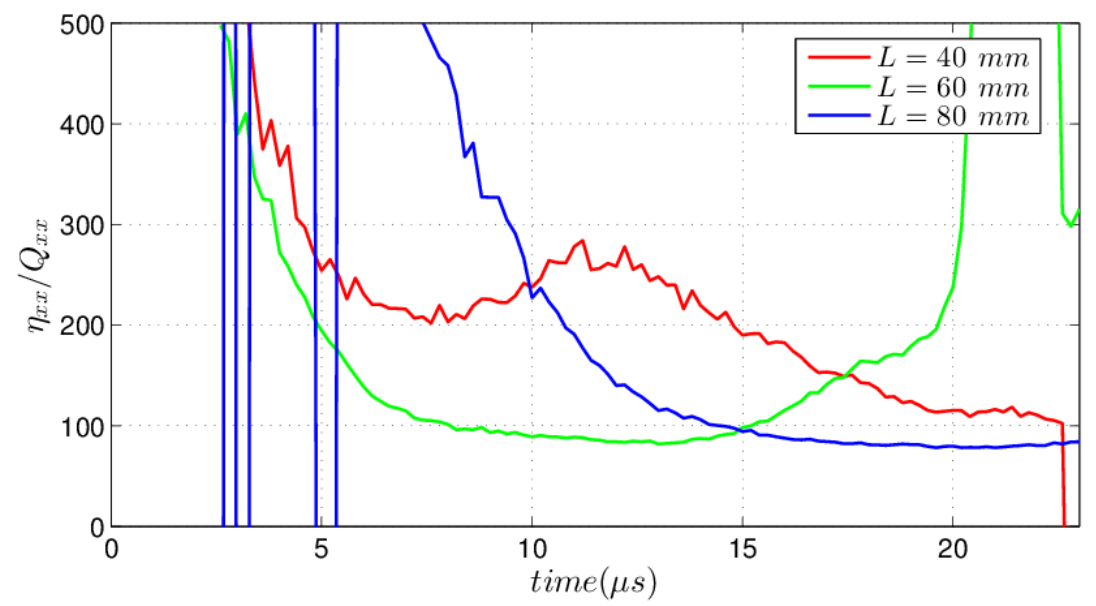

(a)

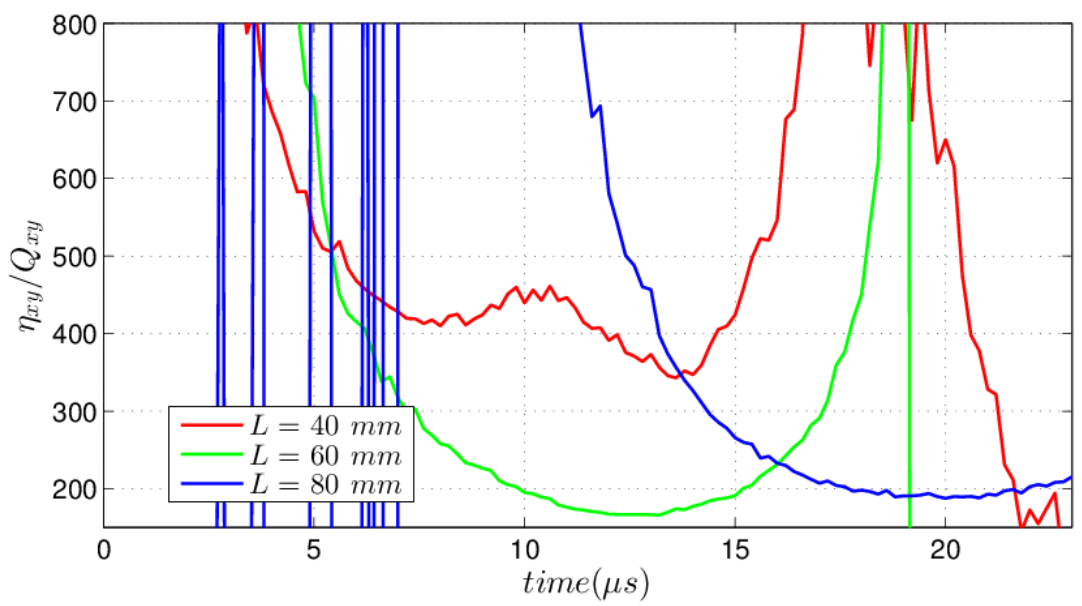

(b)

Figure 22: Coefficients of sensitivity to noise for the three specimens. Virtual mesh: $m=13, n=3$. (a) $\eta_{x x}$. (b) $\eta_{x y}$. 
Table 1: Dimensions, impact speeds and region of interests for the different specimens.

\begin{tabular}{llll}
\hline & Length: $m m$ & Impact speed: $m \cdot s^{-1}$ & Region of Interests \\
Specimen 1 & 40 & $\approx 35$ & Column 6 to 54 \\
Specimen 2 & 60 & $\approx 45$ & Column 5 to 56 \\
Specimen 3 & 80 & $\approx 45$ & Column 4 to 66 \\
\hline
\end{tabular}

Table 2: Imaging and measurement performance information (specimen 1).

\begin{tabular}{ll}
\hline Pixel array size & $400 \times 250$ \\
Inter-frame time (microsecond) & 0.2 \\
Number of images (FP mode) & 128 \\
Exposure time (nanosecond) & 110 \\
\hline Pitch of the grid $(\mathrm{mm})$ & 0.6 \\
Sampling (pixel per period) & 5 \\
Field of view $(\mathrm{mm})$ & $33.6 \times 26.4$ \\
Raw displacement resolution & $0.17 \%$ of grid pitch $(0.0085$ pixel) \\
Displacement spatial smoothing & Gaussian, $3 \times 3$ then $10 \times 10$ data points \\
Displacement temporal smoothing & $3^{r d}$ order polynomial over 25 images \\
Strain temporal smoothing & $3^{r d}$ order polynomial over 25 images \\
Strain resolution (microstrain) & 34 \\
Acceleration resolution $\left(m . s^{-2}\right)$ & $1.4 \times 10^{4}$ \\
\hline
\end{tabular}

Table 3: Details of the ABAQUS/EXPLICIT model.

\begin{tabular}{ll}
\hline Mesh size $(\mathrm{mm})$ & 0.5 \\
Element type & $C 3 D 8 R^{*}$ \\
Time step (microsecond) & Automatic (around 0.01) \\
Inter-frame time (microsecond) & 1 \\
Contact type & Hard contact \\
Impact speed $\left(m \cdot \mathrm{s}^{-1}\right)$ & 30 \\
\hline${ }^{*}$ CPS8R: 8-node linear brick, reduced integration, hourglass control.
\end{tabular}

Table 4: Identification of $E$ and $\nu$ from the large over-determined system with data from the central area. $\mathrm{C}(*)$ means contact model $(*)$. Reference: $E=47.5$ GPa. $\nu=0.3$.

\begin{tabular}{|c|c|c|c|c|c|c|}
\hline & \multicolumn{3}{|c|}{$E: G P a$} & \multicolumn{3}{|c|}{$\nu$} \\
\hline & Top & Bottom & Average field & Top & Bottom & Average field \\
\hline $40 \mathrm{~mm}, \mathrm{C}(1)$ & $47.9(0.8 \%)$ & $48.0(1.1 \%)$ & $46.9(1.3 \%)$ & $0.31(3.3 \%)$ & $0.31(3.3 \%)$ & $0.29(3.3 \%)$ \\
\hline $40 \mathrm{~mm}, \mathrm{C}(2)$ & $38.0(20.0 \%)$ & $41.5(12.6 \%)$ & $46.9(1.3 \%)$ & $0.02(93.3 \%)$ & $0.10(66.7 \%)$ & $0.29(3.3 \%)$ \\
\hline $60 \mathrm{~mm}, \mathrm{C}(2)$ & $46.2(2.7 \%)$ & $48.6(2.3 \%)$ & $47.3(0.4 \%)$ & $0.22(26.7 \%)$ & $0.35(16.7 \%)$ & $0.30(0.0 \%)$ \\
\hline $80 \mathrm{~mm}, \mathrm{C}(3)$ & $48.3(1.7 \%)$ & $49.4(4.0 \%)$ & $47.4(0.2 \%)$ & $0.34(13.3 \%)$ & $0.44(46.7 \%)$ & $0.30(0.0 \%)$ \\
\hline
\end{tabular}


Table 5: Identification from the large over-determined system with experimental data for the three specimens. $E_{Q S}=47.1$ GPa.

\begin{tabular}{lll}
\hline & E: GPa & $\nu$ \\
Specimen 1 $(\mathrm{L}=40 \mathrm{~mm})$ & 70.6 & 0.26 \\
Specimen 2 $(\mathrm{L}=60 \mathrm{~mm})$ & 50.8 & -0.14 \\
Specimen 3 $(\mathrm{L}=80 \mathrm{~mm})$ & 51.9 & -0.10 \\
\hline
\end{tabular}

